\title{
BRUNO BLIND
}

\section{Distributions homogènes sur une algèbre de Jordan}

Bulletin de la S. M. F., tome 125, no 4 (1997), p. 493-528

<http://www.numdam.org/item?id=BSMF_1997_125_4_493_0>

C B Bulletin de la S. M. F., 1997, tous droits réservés.

L'accès aux archives de la revue «Bulletin de la S. M. F. » (http: //smf.emath.fr/Publications/Bulletin/Presentation.html) implique l'accord avec les conditions générales d'utilisation (http://www.numdam.org/ conditions). Toute utilisation commerciale ou impression systématique est constitutive d'une infraction pénale. Toute copie ou impression de ce fichier doit contenir la présente mention de copyright.

\section{Numdam}


Bull. Soc. math. France, 125,1997 , p. $493-528$.

\title{
DISTRIBUTIONS HOMOGÈNES SUR UNE ALGÈBRE DE JORDAN
}

\author{
PAR BRUNO BLIND $(*)$
}

«Gute Uhrwerke sind sie : nur sorge man, sie richtig aufzuziehn!...»

(«Ce sont de bonnes horloges; qu'on prenne soin seulement de les bien remonter !...»)

F. Nietzsche : Also sprach Zarathustra

\begin{abstract}
RÉSUMÉ. - Nous donnons une base holomorphe (en $s$ ) de l'espace $\mathcal{H}^{s}$ des distributions homogènes de degré $s$ sur une algèbre de Jordan $V$ euclidienne simple, et établissons pour la transformée de Fourier de cette base des formules qui constituent la généralisation directe des formules classiques donnant la transformée de Fourier des distributions $|x|^{s}$ et $\operatorname{sign}(x)|x|^{s}$ sur $\mathbb{R}$.

ABSTRACT. - We give a holomorphic (in $s$ ) basis of the space $\mathcal{H}^{s}$ of $s$-homogeneous distributions on simple euclidean Jordan algebras. We compute also the Fourier transform of this basis. This gives a direct generalization of classical formulas for the Fourier transform of the distributions $|x|^{s}$ and $\operatorname{sign}(x)|x|^{s}$.
\end{abstract}

\section{Introduction}

Soit $V$ un espace vectoriel de dimension finie sur lequel agit un groupe $G$; la détermination des distributions homogènes sous l'action du groupe $G$ est un problème classique ( $c f$. A. Weil [We] et M. Raïs [Ra]).

Dans [Ri-St], F. Ricci et E.M. Stein donnent la dimension de l'espace $\mathcal{H}^{s}$ des distributions $G$-homogènes de degré $s$ sur l'espace $\operatorname{Herm}(r, \mathbb{C})$ où $G$ est

(*) Texte reçu le 11 juillet 1996, révisé le 17 juillet 1997, accepté le 14 novembre 1997. B. BLIND, URA CNRS 750, Département de Mathématiques, Université H. Poincaré, BP 239, 54506 Vandoeuvre-lés-Nancy. Email : blind@iecn.u-nancy.fr.

Classification AMS : 46 F 10, $17 \mathrm{C}$.

Mots clés : Distributions homogènes, algèbres de Jordan, distribution zêta, transformation de Fourier.

BULlETiN DE LA SOCIÉtÉ MATHÉMATIQUE DE FRANCE 0037-9484/1997/493/\$5.00

(C) Société mathématique de France 
le groupe $\mathrm{GL}(r, \mathbb{C})$ : la dimension est égale au nombre d'orbites ouvertes du groupe $G$, soit $r+1$. Ces auteurs évoquent la possibilité d'étendre leur méthode au cas d'une algèbre de Jordan simple et euclidienne; M. Muro, utilisant l'analyse micro-locale, donne la dimension de l'espace $\mathcal{H}^{s}$ dans ce cas $(c f .[\mathrm{Mu}]$, elle est toujours égale au nombre d'orbites ouvertes $\mathrm{du}$ groupe $G)$.

Nous montrons dans ce travail que les techniques de [Ri-St], généralisées aux algèbres de Jordan simples et euclidiennes, et les résultats de J. Faraut et I. Satake sur les coefficients intervenant dans l'équation fonctionnelle des distributions zêta conduisent naturellement à une base de l'espace $\mathcal{H}^{s}$ qui constitue un analogue des distributions $|x|^{s}$ et $\operatorname{sign}(x)|x|^{s}$ sur $\mathbb{R}$.

Après les rappels sur les algèbres de Jordan, nous généralisons dans le paragraphe 3 aux algèbres de Jordan simples et euclidiennes, certains résultats de F. Ricci et E.M. Stein sur les pôles, ainsi que sur le support des coefficients intervenant dans la partie singulière du développement de Laurent en ces points, des distributions du type

$$
\sum_{i=0}^{r} a_{i}|\operatorname{det} x|^{s} \chi_{\Omega_{i}}(x)
$$

où les coefficients $a_{i}$ sont dans $\mathbb{C}$. Notons que M. Muro [Zürich, 1994] trouve, en utilisant l'analyse micro-locale, les mêmes résultats dans le cas de l'algèbre des matrices symétriques réelles.

Ces résultats, combinés avec ceux de M. Muro [Mu] sur la dimension de l'espace $\mathcal{H}^{s}$, nous permettent de donner, au paragraphe 4 , une base holomorphe en $s$ de $\mathcal{H}^{s}$. Nous calculons également la transformée de Fourier de cette base et trouvons des formules analogues à celles que I.M. Gelfand et G.E. Chilov établissent pour $|x|^{s}$ et $\operatorname{sign}(x)|x|^{s}$ (voir [Ge-Ch, formules $12^{\prime}$ et 13 , p. 170-171]) :

$$
\hat{f}_{s}=\sqrt{2 \pi} f_{-s-1}, \quad \hat{g}_{s}=i \sqrt{2 \pi} g_{-s-1}
$$

où

$$
f_{s}(x)=2^{-\frac{1}{2} s} \frac{|x|^{s}}{\Gamma\left(\frac{1}{2}(s+1)\right)}, \quad g_{s}(x)=2^{-\frac{1}{2} s} \frac{\operatorname{sign}(x)|x|^{s}}{\Gamma\left(\frac{1}{2}(s+2)\right)} .
$$

Avec nos théorèmes 3.3 et 3.6 , on peut copier la méthode de $\mathrm{F}$. Ricci et E.M. Stein et redémontrer le résultat de M. Muro sur la dimension de l'espace $\mathcal{H}^{s}$, c'est ce que nous indiquons brièvement dans l'appendice.

C'est avec plaisir que je remercie J. Faraut pour m'avoir suggéré le problème, et P.Y. Gaillard pour ses nombreux encouragements. Je remercie également le rapporteur de la première version de ce travail pour m'avoir (fortement) incité à rajouter le paragraphe 6 .

TOME $125-1997-\mathrm{N}^{\circ} 4$ 


\section{Notations et rappels sur les algèbres de Jordan}

\section{A. Les algèbres de Jordan simples et euclidiennes.}

Nous renvoyons à [Br-Koe], [Fa-Ko], [Sa1] pour les définitions et les principaux faits concernant les algèbres de Jordan.

Soit $V$ une algèbre de Jordan euclidienne sur le corps $\mathbb{R}$, simple, de dimension $n$, de rang $r$ et d'élément unité $e$. L'entier $d$ est déterminé par l'égalité

$$
n=r+\frac{1}{2} d r(r-1) .
$$

Pour $x$ et $y$ dans $V$, on définit les endomorphismes de $V$ :

$$
\mathcal{L}(x) y=x y, \mathcal{P}(x)=2 \mathcal{L}^{2}(x)-\mathcal{L}\left(x^{2}\right), x \square y=\mathcal{L}(x y)+[\mathcal{L}(x), \mathcal{L}(y)] .
$$

On munira $V$ de la forme bilinéaire définie positive

$$
\langle x, y\rangle=\frac{r}{n} \operatorname{tr} \mathcal{L}(x y)
$$

et $\mathrm{d} x$ désignera la mesure euclidienne associée à ce produit scalaire.

On note det $x$ le déterminant de $V$. C'est un polynôme irréductible et homogène de degré $r$. Un élément $x$ de $V$ est inversible si det $x \neq 0$. Nous noterons Det $g$ le déterminant d'un élément $g$ de $\mathrm{GL}(V)$.

Tout élément $x$ de l'algèbre $V$ peut s'écrire sous la forme

$$
x=\sum_{i=1}^{r} \lambda_{i} e_{i}, \quad \lambda_{i} \in \mathbb{R},
$$

où $\left\{e_{1}, \ldots, e_{r}\right\}$ est un système complet d'idempotents primitifs orthogonaux (dépendant de $x$ ). Les nombres $\lambda_{i}$ sont appelés les valeurs propres de $x$ et l'on a

$$
\operatorname{det} x=\prod_{i=1}^{r} \lambda_{i} .
$$

Le rang de $x$ est le nombre de réels $\lambda_{i}$ non nuls (comptés avec multiplicité). Si un élément inversible $x$ possède $p$ valeurs propres positives et $q$ valeurs propres négatives, on dira que sa signature est $(p, q)(p+q=r)$. On note $\Omega_{j}$ l'ensemble des éléments inversibles de signature $(r-j, j)$. Le cône $\Omega=\Omega_{0}$ est la composante connexe contenant $e$ de l'ensemble des éléments inversibles de $V$.

Notons $G$ la composante neutre du groupe de structure de $V$ et fixons un système complet d'idempotents orthogonaux $\left\{e_{1}, \ldots, e_{r}\right\}$ de l'algèbre $V$; le groupe $G$ admet une décomposition d'Iwasawa $G=K A N$ 
où $K$ est le sous-groupe de $G$ des éléments qui fixent l'unité $e, A$ est engendré par les $\mathcal{P}\left(\sum_{i=1}^{r} a_{i} e_{i}\right)$ (avec $a_{i}>0$ ), et où $N$ est engendré par les transformations de Frobenius $\exp \left(2 z \square e_{i}\right)$ avec $z$ dans

$$
V_{i j}=\left\{x \in V ; e_{i} x=e_{j} x=\frac{1}{2} x\right\}, \quad i<j,
$$

(pour tout ceci voir [Fa-Ko]). Les orbites de $V$ sous l'action du groupe $G$ ont été déterminées par S. Kaneyuki et I. Satake (cf $[\mathrm{Ka}],[\mathrm{Sa} 2])$ : ce sont les ensembles $S_{p, q}=G o_{p, q}$ où

$$
o_{p, q}=\sum_{i=1}^{p-q} e_{i}-\sum_{i=1}^{q} e_{p-q+i} \quad(\operatorname{avec} 0 \leq q \leq p \leq r)
$$

en particulier la signature est invariante sous $G$. Pour $p=r$, les orbites $S_{p, q}$ sont ouvertes et l'on a $S_{r, q}=\Omega_{q}$; les autres orbites sont contenues dans le lieu singulier $S=\{x \in V$; det $x=0\}$ de l'algèbre $V$ et sont en fait des $G^{1}$ orbites où $G^{1}=\{g \in G ; \operatorname{Det} g=1\}$.

Rappelons brièvement la classification des algèbres de Jordan sur $\mathbb{R}$ simples et euclidiennes (voir [Br-Koe], [Fa-Ko]).

1) $V=\operatorname{Sym}(r, \mathbb{R})$ l'espace des matrices symétriques réelles d'ordre $r$ avec

$$
x \cdot y=\frac{1}{2}(x y+y x), \quad d=1 \quad n=\frac{1}{2} r(r+1), \quad\langle x, y\rangle=\operatorname{tr} x y .
$$

Ici le déterminant est le déterminant usuel.

2) $V=\operatorname{Herm}(r, \mathbb{C})$ l'espace des matrices hermitiennes complexes d'ordre $r$ avec

$$
x \cdot y=\frac{1}{2}(x y+y x), \quad d=2, \quad n=r^{2}, \quad\langle x, y\rangle=\operatorname{tr} x y .
$$

Ici encore le déterminant est le déterminant usuel.

3) $V=\operatorname{Herm}(r, \mathbb{H})$ l'espace des matrices hermitiennes sur $\mathbb{H}$ d'ordre $r$, avec le produit

$$
x \cdot y=\frac{1}{2}(x y+y x) .
$$

Ici $d=4$.

4) $V=\mathbb{R} \times E$ où $E$ est un espace euclidien de produit scalaire $(., .)_{E}$, de dimension $n-1$. Le produit de Jordan est défini par

$$
(\lambda, u)(\mu, v)=\left(\lambda \mu+(u, v)_{E}, \lambda v+\mu u\right) ;
$$

ici $r=2, d=n-2$ et le déterminant est donné par $\operatorname{det}(\lambda, u)=\lambda^{2}-\|u\|_{E}^{2}$.

5) Une algèbre de Jordan exceptionnelle constituée de matrices $(3,3)$ à coefficients dans les octaves de Cayley $(d=8, r=3, n=27)$. 


\section{B. L'équation fonctionnelle des distributions zêta.}

On désigne par $\mathcal{S}(V)$ l'espace de Schwartz de $V$; pour $f$ dans $\mathcal{S}(V)$ les intégrales

$$
\Phi_{j}(f, s)=\int_{\Omega_{j}}|\operatorname{det} x|^{s} f(x) \mathrm{d} x, \quad(\operatorname{Re} s \geq 0)
$$

définissent des distributions tempérées. Ces distributions admettent un prolongement méromorphe sur $\mathbb{C}$, encore noté $\Phi_{j}(., s)$. Cela résulte de l'identité de Bernstein :

$$
(\operatorname{det} \partial)|\operatorname{det} x|^{s}=(-1)^{j} b(s)|\operatorname{det} x|^{s-1}, \quad x \in \Omega_{j},
$$

(avec $b(s)=s\left(s+\frac{1}{2} d\right) \cdots\left(s+\frac{1}{2}(r-1) d\right)$ et où $\operatorname{det} \partial$ est l'opérateur différentiel à coefficients constants tel que $\operatorname{det} \partial \exp (\langle., y\rangle)=\operatorname{det} y \exp (\langle., y\rangle)$.

La fonction gamma du cône $\Omega$,

$$
\Gamma_{\Omega}(s)=\int_{\Omega} \mathrm{e}^{-\langle x, \mathrm{e}\rangle}(\operatorname{det} x)^{s-\frac{n}{r}} \mathrm{~d} x,
$$

a été calculée par S.G. Gindikin [Gi] :

$$
\Gamma_{\Omega}(s)=(2 \pi)^{\frac{1}{2}(n-r)} \prod_{j=0}^{r-1} \Gamma\left(s-\frac{1}{2} j d\right) .
$$

On sait d'après l'identité de Bernstein que, si $s_{0}$ est un pôle de la distribution $\Phi_{j}(., s)$, son ordre $o_{j}\left(s_{0}\right)$ est borné par l'ordre $o\left(s_{0}\right)$ du pôle $s_{0}$ pour la fonction $\Gamma_{\Omega}\left(s+\frac{n}{r}\right)$. Si $s_{0}$ est un pôle effectif de la distribution $\Phi_{j}(., s)$, on note

$$
\sum_{h=1}^{o_{j}\left(s_{0}\right)} \frac{B_{j}^{h}\left(., s_{0}\right)}{\left(s-s_{0}\right)^{h}}
$$

la partie singulière de son développement de Laurent au voisinage de ce point. Les coefficients $B_{j}^{h}\left(., s_{0}\right)\left(1 \leq h \leq o_{j}\left(s_{0}\right)\right)$ de ce développement sont des distributions tempérées, dont le support est contenu dans le lieu singulier $S$ (c'est une réunion de $S_{p, q}$ avec $p<r$ ).

Remarque 2.1. - Notons $\Sigma$ la sphère unité de $V$; on a :

$$
\Phi_{j}(f, s)=c \int_{0}^{\infty} \rho^{r s+n-1} \int_{\Omega_{j} \cap \Sigma}|\operatorname{det} u|^{s} f(\rho u) \mathrm{d} \sigma(u) \mathrm{d} \rho \quad(\operatorname{Re} s>0),
$$

où $c$ est une constante de normalisation. 
Pour Re $s>-n / r$, la fonction $\rho^{r s+n-1}$ est localement intégrable; par conséquent les pôles de $\Phi_{j}(f, s)$ compris dans l'intervalle ] $\left.-n / r,-1\right]$ proviennent de ceux de :

$$
f \mapsto \int_{\Omega_{j} \cap \Sigma}|\operatorname{det} u|^{s} f(u) \mathrm{d} \sigma(u)
$$

et en particulier aucune des distributions $B_{j}^{o_{j}\left(s_{0}\right)}\left(., s_{0}\right)$ n'est portée par l'origine.

Posons

$$
\gamma(s)=\Gamma_{\Omega}(s) \exp \left(-\frac{1}{2} i \pi r s\right)
$$

et définissons la transformée de Fourier d'un élément $f$ de $\mathcal{S}(V)$ par

$$
\widehat{f}(y)=\int_{V} \mathrm{e}^{-i\langle x, y\rangle} f(x) \mathrm{d} x
$$

on a l'équation fonctionnelle (voir [Sa-Sh]) :

ThÉoRÈme 2.1. - La transformée de Fourier de la distribution $\Phi_{j}(., s)$ est donnée par:

$$
\Phi_{j}\left(\widehat{f}, s-\frac{n}{r}\right)=\gamma(s) \sum_{k=0}^{r} u_{j k}(s) \Phi_{k}(f,-s)
$$

où les fonctions $u_{j k}(s)$ sont des polynômes en $\exp (i \pi s)$.

Remarque 2.2. - Dans [Sa-Fa], la transformée de Fourier d'un élément $f$ de $\mathcal{S}(V)$ est définie par

$$
\widehat{f}(y)=\int_{V} e^{2 i \pi\langle x, y\rangle} f(x) \mathrm{d} x
$$

d'autre part, ces auteurs mettent le terme $\Gamma_{\Omega}(s) \exp \left(\frac{1}{2} i \pi r s\right)$ (alors que nous mettons le terme $\Gamma_{\Omega}(s) \exp \left(-\frac{1}{2} i \pi r s\right)$ ) en facteur dans le membre de droite de l'équation fonctionnelle. Compte tenu de ces différences, les coefficients notés $u_{j k}(s)$ dans [Sa-Fa] sont ici $\exp (-i \pi r s) u_{r-j k}(s)$.

Les fonctions $u_{j k}(s)$ sont explicitement connues : pour $k$ compris entre 0 et $r$, posons

$$
P_{k}(\mathrm{X})=\prod_{p=1}^{k}\left(1+(-1)^{d(k-p)} \mathrm{X}\right) \quad\left(P_{0}=1\right)
$$

on a $(c f .[\mathrm{Sa}-\mathrm{Fa}])$ :

$$
\text { TOME } 125-1997-\mathrm{N}^{\circ} 4
$$


Proposition 2.2. - Posons $q=\exp (i \pi s)$. On a l'égalité :

$$
\begin{aligned}
\sum_{j=0}^{r} \mathrm{X}^{j} u_{j k}(s)=q^{k} P_{r-k}\left(\operatorname { e x p } \left(-\frac{1}{2} i\right.\right. & \pi d(r-1)) \mathrm{X} q) \\
\times & P_{k}\left(\exp \left(\frac{1}{2} i \pi d(r-1)\right) \mathrm{X} q^{-1}\right) .
\end{aligned}
$$

La formulation de cette proposition m'a été communiquée par J. Faraut; comme elle ne se trouve pas sous cette forme dans [Sa-Fa], donnons quelques indications sur sa démonstration. On part de la formule (25), p. 479 de $[\mathrm{Sa}-\mathrm{Fa}]$; dans cette formule on remplace $y$ par $y^{-1}$ puis on multiplie par $y^{r}$. Il vient dans nos notations ( $c f$. la remarque 2.2!) :

$$
\begin{aligned}
& \sum_{i=0}^{r} y^{i} u_{i j}(s)=q^{r} \prod_{k=1}^{j}\left((-1)^{d k} i^{d(r+1)} q^{-1} y+1\right) \\
& \times \prod_{k=1}^{r}\left((-1)^{d(k-j)} i^{-d(r+1)} y+q^{-1}\right) .
\end{aligned}
$$

On vérifie alors que le premier et le deuxième produits du membre de droite sont respectivement

$$
P_{j}\left(\exp \left(\frac{1}{2} i \pi d(r-1)\right) y q^{-1}\right), \quad q^{-(r-j)} P_{r-j}\left(\exp \left(-\frac{1}{2} i \pi d(r-1)\right) y q\right) .
$$

Donnons quelques conséquences de cette proposition lorsque l'entier $d$ est pair. Dans ce cas $n / r=1+\frac{1}{2} d(r-1)$ est entier, $P_{k}(\mathrm{X})=(1+\mathrm{X})^{k}$, et

$$
\sum_{j=0}^{r} \mathrm{X}^{j} u_{j k}(s)=\left(1+(-1)^{\frac{1}{2} d(r-1)} \mathrm{X} q\right)^{r-k}\left(q+(-1)^{\frac{1}{2} d(r-1)} \mathrm{X}\right)^{k} .
$$

I. Satake et J. Faraut exploitent cette relation en interprétant la matrice $U(s)=\left(u_{j k}(s)\right)$ comme matrice d'une représentation du groupe $\mathrm{GL}_{2}(\mathbb{C})$. Soit $\rho_{r}$ la représentation de $\mathrm{GL}_{2}(\mathbb{C})$ dans l'espace $\mathcal{P}_{r}$ des polynômes à une variable, de degré inférieur ou égal à $r$, définie par

$$
\rho_{r}\left(\begin{array}{ll}
a & b \\
c & d
\end{array}\right) f(\mathrm{X})=(b \mathrm{X}+d)^{r} f\left(\frac{a \mathrm{X}+c}{b \mathrm{X}+d}\right) .
$$

On a :

$$
\begin{aligned}
\rho_{r}\left(\begin{array}{cc}
1 & q \\
q & 1
\end{array}\right) \mathrm{X}^{k} & =(1+\mathrm{X} q)^{r-k}(q+\mathrm{X})^{k}, \\
\rho_{r}\left(\begin{array}{cc}
1 & q \\
-q & -1
\end{array}\right) \mathrm{X}^{k} & =(-1)^{r}(1-\mathrm{X} q)^{r-k}(q-\mathrm{X})^{k} .
\end{aligned}
$$


Alors $U(s)$ est la matrice dans la base canonique de $\mathcal{P}_{r}$ de l'opérateur $\rho_{r}\left(\begin{array}{ll}1 & q \\ q & 1\end{array}\right)$ si $\frac{n}{r}$ est impair, et de $\rho_{r}\left(\begin{array}{cc}1 & q \\ -q & -1\end{array}\right)$ si $\frac{n}{r}$ est pair.

Il en résulte (cf. [Sa-Fa, p. 481]) qu'il existe une famille de distributions $\left\{\Psi_{i}(., s) ; 0 \leq i \leq r\right\}$

$$
\Psi_{i}(., s)=\sum_{j=0}^{r} a_{i j} \Phi_{j}(., s),
$$

où les coefficients $a_{i j}$ sont déterminés par les relations

$$
\sum_{i=0}^{r} \mathrm{X}^{i} a_{i j}=\rho_{r}\left(\begin{array}{cc}
1 & 1 \\
1 & -1
\end{array}\right) \mathrm{X}^{j}=(\mathrm{X}-1)^{r-j}(\mathrm{X}+1)^{j}
$$

avec :

$$
\widehat{\Psi}_{i}\left(., s-\frac{n}{r}\right)= \begin{cases}\gamma(s)(1-q)^{r-i}(1+q)^{i} \Psi_{i}(.,-s) & \text { si } n / r \equiv 1 \bmod 2 \\ \gamma(s)(1+q)^{r-i}(1-q)^{i} \Psi_{r-i}(.,-s) & \text { si } n / r \equiv 0 \bmod 2 .\end{cases}
$$

Ce sont ces distributions $\Psi_{i}(., s)$ qui vont constituer les analogues des distributions $|x|^{s}$ et $\operatorname{sign}(x)|x|^{s}$.

Remarque 2.3. - Notons que

$$
\Psi_{r}(., s)=|\operatorname{det} x|^{s} \quad \text { et } \quad \Psi_{0}(., s)=(-1)^{r}|\operatorname{det} x|^{s} \operatorname{sign}(\operatorname{det} x) .
$$

Remarquons d'autre part que la famille de distributions

$$
\left\{\Psi_{i}(., s) / 0 \leq i \leq r\right\}
$$

est libre pour $s \neq-k, k$ entier plus grand ou égal à 1 .

Remarque 2.4.- Nos distributions $\Psi_{i}(., s)$ sont les distributions notées $\Phi_{i}^{\prime}(., s)$ dans [Sa-Fa, p. 481]. Noter à ce sujet que la formule (30), p. 481, a été dénaturée par une erreur d'impression signalée dans [Sa-Og, p. 12].

Dans le cas où l'entier $d$ est impair, la situation est un peu plus difficile à décrire; pour ne pas alourdir l'exposé, nous donnerons les détails dans le paragraphe 5 .

DÉfinition 2.3. - Une distribution $T$ sur $V$ est dite $G$-homogène de degré $s$ si

$$
\left\langle T, \phi_{g}\right\rangle=(\operatorname{Det} g)^{\frac{r s}{n}}+1\langle T, \phi\rangle
$$

où $g$ est dans $G$ et avec $\phi_{g}(x)=\phi\left(g^{-1} x\right)$. On notera $\mathcal{H}^{s}$ l'espace des distributions $G$-homogènes de degré $s$. 
Les distributions $\Phi_{i}(., s)$, quand elles existent, sont des éléments de l'espace $\mathcal{H}^{s}$ et si $s_{0}$ est un pôle de $\Phi_{i}(., s)$, le coefficient $B_{i}^{o_{i}\left(s_{0}\right)}\left(., s_{0}\right)$ est dans $\mathcal{H}^{s}$, puisque $\left(s-s_{0}\right)^{o_{i}\left(s_{0}\right)} \Phi_{i}(., s)$ tend vers $B_{i}^{o_{i}\left(s_{0}\right)}\left(., s_{0}\right)$ quand $s$ tend vers $s_{0}$. M. Muro [Mu] a démontré que dans le cas d'une algèbre de Jordan simple et euclidienne, l'espace vectoriel $\mathcal{H}^{s}$ est de dimension $r+1$.

\section{3. Étude des combinaisons linéaires de distributions $\Phi_{i}(., s)$}

Le but de ce paragraphe est de généraliser les résultats de $\mathrm{F}$. Ricci et E. Stein concernant les distributions qui sont combinaisons linéaires des distributions $\Phi_{i}(., s)$ (ces auteurs se placent dans le cadre des matrices hermitiennes $\operatorname{Herm}(r, \mathbb{C}))$.

\section{A. Une réduction au rang $r-1$.}

Fixons un système complet d'idempotents orthogonaux $\left\{e_{1}, \ldots, e_{r}\right\}$ et considérons la décomposition de Peirce associée à ce système :

$$
V=\bigoplus_{1 \leq i \leq j \leq r} V_{i j} .
$$

(Pour un élément $x$ de $\mathrm{V}$, on désigne par $x_{i j}$ les composantes de cet élément suivant cette décomposition.) L'algèbre

$$
V^{\prime}=\left\{x \in V ; e_{1} x=0\right\}=\bigoplus_{2 \leq i \leq j \leq r} V_{i j}
$$

est une sous-algèbre de $V$, simple, euclidienne et de rang $r-1$. Dans la suite, si $O$ est un objet relatif à l'algèbre $V$, nous noterons $O^{\prime}$ l'objet correspondant pour l'algèbre $V^{\prime}$.

Posons

$$
W=\left\{x \in V ; e_{1} x=\frac{1}{2} x\right\}=\bigoplus_{2 \leq j \leq r} V_{1 j}
$$

et considérons l'application $\Phi$ suivante :

$$
\begin{aligned}
& \Phi: \mathbb{R} \times W \times V^{\prime} \longrightarrow V, \\
& \quad(u, z, v) \longmapsto \exp \left(2 z \square e_{1}\right)\left(u e_{1}+v\right) .
\end{aligned}
$$

Proposition 3.1. - L'application $\Phi$ réalise un $C^{\infty}$ difféomorphisme de $\mathbb{R}^{*} \times W \times V^{\prime}$ sur l'ouvert $U=\left\{x \in V ; x_{11} \neq 0\right\}$. De plus, on a

$$
\Phi\left(\mathbb{R}^{*+} \times W \times \Omega_{i}^{\prime} \cup \mathbb{R}^{*-} \times W \times \Omega_{i-1}^{\prime}\right)=\Omega_{i} \cap U
$$

pour $0<i<r$ ainsi que

$$
\Phi\left(\mathbb{R}^{*+} \times W \times \Omega_{0}^{\prime}\right)=\Omega_{0} \cap U, \quad \Phi\left(\mathbb{R}^{*-} \times W \times \Omega_{r-1}^{\prime}\right)=\Omega_{r} \cap U .
$$


En utilisant le lemmme VI.3.1, p. 106, de [Fa-Ko], on vérifie que

$$
\Phi(u, z, v)=u e_{1}+u z+v+u\left(e-e_{1}\right)(z \cdot z) .
$$

Il n'est pas difficile de voir que $\left(e-e_{1}\right) \cdot(z \cdot z)$ appartient à $V^{\prime}$ (en fait, $2\left(e-e_{1}\right) \cdot(z \cdot z)$ est la projection sur $V^{\prime}$ de $z \cdot z$; pour toutes ces proprités de la décomposition de Peirce de $V$, voir [Fa-Ko]). Il en résulte l'injectivité de l'application $\Phi$. Soit d'autre part $x=u e_{1}+z+v\left(z \in W, v \in V^{\prime}\right.$, $u \neq 0$ ) un élément de l'ouvert $U$; toujours par le lemme VI.3.1 de [Fa-Ko], on voit que

$$
\Phi\left(u, \frac{z}{u}, v-\frac{1}{u}(z \cdot z)_{V^{\prime}}\right)=x
$$

(où $(z \cdot z)_{V^{\prime}}$ désigne la projection sur $V^{\prime}$ de $z \cdot z$ dans la décomposition de Peirce), ce qui donne la surjectivité de $\Phi$.

D'autre part, comme $\exp \left(2 z \square e_{1}\right)$ appartient à $G$, il conserve la signature d'un élément de $V$; on en déduit la proposition.

RemARque 3.1. - On a une proposition analogue pour les orbites singulières $S_{p, q}$.

Soit $T$ une distribution sur $U$; le difféomorphisme $\Phi$ transporte $T$ en une distribution sur l'ensemble $\mathbb{R}^{*} \times W \times V^{\prime}$ notée $\Phi^{*} T$. Notons $\Phi_{i}^{U}(., s)$ la restriction à l'ouvert $U$ de la distribution $\Phi_{i}(., s)$. Pour $s$ dans $\mathbb{C}$ définissons les distributions $x_{+}^{s}$ (resp. $x_{-}^{s}$ ) par :

$$
x_{+}^{s}(\phi)=\int_{0}^{\infty} x^{s} \phi(x) \mathrm{d} x \quad\left(\text { resp. } x_{-}^{s}(\phi)=\int_{-\infty}^{0}|x|^{s} \phi(x) \mathrm{d} x\right)
$$

où $\phi$ est une fonction $C^{\infty}$ dans $\mathbb{R}^{*}$ à support compact.

Corollaire 3.2. - En tant que fonctions méromorphes de s, on a

$$
\Phi^{*} \Phi_{i}^{U}(., s)=x_{-}^{s+d(r-1)} \otimes \mathrm{d} z \otimes \Phi_{i-1}^{\prime}(., s)+x_{+}^{s+d(r-1)} \otimes \mathrm{d} z \otimes \Phi_{i}^{\prime}(., s)
$$

pour $0<i<r$ ainsi que

$$
\begin{aligned}
& \Phi^{*} \Phi_{0}^{U}(., s)=x_{+}^{s+d(r-1)} \otimes \mathrm{d} z \otimes \Phi_{0}^{\prime}(., s), \\
& \Phi^{*} \Phi_{r}^{U}(., s)=x_{-}^{s+d(r-1)} \otimes \mathrm{d} z \otimes \Phi_{r-1}^{\prime}(., s) .
\end{aligned}
$$

Soit $\phi$ une fonction test à support inclus dans l'ouvert $U$. Dans l'intégrale définissant $\Phi_{i}(\phi, s)$ pour $\operatorname{Re} s>0$, faisons le changement de 
variable $x=\Phi(u, z, v)$; son jacobien est $\mathrm{d} x=|u|^{d(r-1)} \mathrm{d} u \mathrm{~d} z \mathrm{~d} v$ et par conséquent :

$$
\Phi_{i}(\phi, s)=\Phi_{i}^{\prime}\left(G^{+}, s\right)+\Phi_{i-1}^{\prime}\left(G^{-}, s\right)
$$

avec (où $\epsilon= \pm$ )

$$
G^{\epsilon}(v, s)=\int_{0}^{\infty} \int_{W} \phi \circ \Phi(\epsilon u, z, v) \mathrm{d} z u^{s+d(r-1)} \mathrm{d} u .
$$

Compte tenu de l'hypothèse faite sur le support de la fonction $\phi$, les fonctions $G^{\epsilon}(v, s)$ sont analytiques en $s, C^{\infty}$ et à support compact en $v$. On prolonge alors la relation précédente d'une manière méromorphe à $\mathbb{C}$ tout entier, d'où le corollaire.

REMARQUe 3.2. - Notons que si $s_{0}$ est un pôle effectif de la distribution $\Phi_{i}(., s)$, la fonction $s \mapsto \Phi_{i}^{U}(., s)$ ne pourra se prolonger au point $s_{0}$ que si les distributions $B_{i}^{h}\left(., s_{0}\right)$ sont portées par l'origine pour $1 \leq h \leq o_{i}\left(s_{0}\right)$; cela résulte du fait que l'hyperplan $x_{11}=0$ ne contient que la seule $G^{1}$-orbite $\{0\}$.

\section{B. Généralisation des résultats de F. Ricci et E.M. Stein, le cas $d$ pair.}

On considère une distribution $A(., s)$ de la forme

$$
A(., s)=\sum_{j=0}^{r} c_{j} \Phi_{j}(., s)
$$

Pour un pôle effectif $s_{0}$ d'ordre $\alpha$ de cette distribution, on note

$$
\sum_{h=1}^{h=\alpha} \frac{A^{h}\left(., s_{0}\right)}{\left(s-s_{0}\right)^{h}}
$$

la partie singulière de son développement de Laurent au voisinage de ce pôle. Rappelons d'autre part que nous avons noté $o\left(s_{0}\right)$ l'ordre du pôle $s_{0}$ pour la fonction $\Gamma_{\Omega}(s+n / r)$.

Pour la commodité du lecteur nous avons choisi d'énoncer et de démontrer nos résultats d'abord dans le cas où l'entier $d$ est pair, les démonstrations pour le cas $d$ impair sont essentiellement les mêmes, les calculs étant plus pénibles (voir le paragraphe 5 ).

Les pôles possibles de la distribution $A(., s)$ sont alors les entiers négatifs plus petits que -1 ; à partir d'un tel entier $-k$ et des coefficients $c_{i}$ 
de la distribution $A(., s)$, construisons le polynôme $P$ satisfaisant aux deux conditions suivantes :

$$
\left\{\begin{array}{l}
P(i)=c_{i}(-1)^{k i} \\
\mathrm{~d}^{\circ} P \leq r
\end{array}\right.
$$

ThÉonÈme 3.3. - La distribution $A(., s)$ possède en $s=-k$ un pôle d'ordre

$$
\min \left(\mathrm{d}^{\circ} P, o(-k)\right)
$$

Il en résulte immédiatement le

Corollaire 3.4. - Pour tout $j$ tel que $0 \leq j \leq r$ et tout entier $k$ tel que $k \geq 1$, on $a o_{j}(-k)=o(-k)$.

La démonstration, copiée sur celle du théorème 1 de [Ri-St], se fait en deux étapes.

On suppose d'abord que $k \geq n / r$; alors $o(-k)=r$ et donc $\min \left(\mathrm{d}^{\circ} P, o(-k)\right)=\mathrm{d}^{\circ} P$. Il suffit donc de prouver le résultat ( $k$ étant fixé) pour une base $\left\{P_{0}, \ldots, P_{r}\right\}$ des polynômes de degré inférieur ou égal à $r$, et pour les distributions correspondantes

$$
A_{i}(., s)=\sum_{j=0}^{r} P_{i}(j)(-1)^{k j} \Phi_{j}(., s)
$$

On prend pour polynômes $P_{i}$ les polynômes définis par (les coefficients $a_{i j}$ sont définis au $\S 2$ ) :

$$
\left\{\begin{array}{l}
P_{i}(j)=a_{i j}(-1)^{k j} \\
\mathrm{~d}^{\circ} P_{i} \leq r
\end{array}\right.
$$

Les distributions $A_{i}(., s)$ correspondantes sont alors les distributions $\Psi_{i}(., s)$ définies au paragraphe 2 .

Utilisant les propriétés des nombres $a_{i j}$, il n'est pas difficile de voir que

$$
\mathrm{d}^{\circ} P_{i}= \begin{cases}r-i & \text { si } k \text { est pair } \\ i & \text { sinon. }\end{cases}
$$

Admettons ce résultat pour le moment.

Distinguons deux cas :

- le cas (i) où l'entier $n / r$ est impair;

- le cas (p) où il est pair.

TOME $125-1997-\mathrm{N}^{\circ} 4$ 
Rappelons que pour les distributions $\Psi_{i}(., s)$, on a :

$$
\widehat{\Psi}_{i}(., s)=\left\{\begin{array}{lc}
c_{i}(s) \Psi_{i}\left(.,-s-\frac{n}{r}\right) & \operatorname{cas}(\mathrm{i}), \\
c_{i}(s) \Psi_{r-i}\left(.,-s-\frac{n}{r}\right) & \operatorname{cas}(\mathrm{p}),
\end{array}\right.
$$

avec $c_{i}(s)=\gamma(s+n / r)(1+q)^{r-i}(1-q)^{i}$.

Étudier $\Psi_{i}(., s)$ au point $s=-k$ revient à étudier $\widehat{\Psi}_{i}(., s)$ au même point; d'autre part, pour $k \geq n / r$, les distributions $\Psi_{i}(., k-n / r)$ sont des fonctions localement intégrables, non nulles; il suffit donc d'étudier l'ordre du pôle $-k$ pour la fonction $c_{i}(s)$, ce qui est facile par inspection.

Pour terminer la première étape de la démonstration, il reste à justifier notre assertion en ce qui concerne les polynômes $P_{i}$. Étant donnés $r+1$ nombres $c_{0}, \ldots, c_{r}$, posons

$$
\Delta\left(c_{j}\right)=c_{j+1}-c_{j}, \quad j=0, \ldots, r-1,
$$

et pour $\ell \leq r$, posons

$$
\Delta^{\ell}\left(c_{j}\right)=\Delta\left(\Delta^{\ell-1} c_{j}\right), \quad j=0, \ldots, r-\ell .
$$

Un résultat classique d'interpolation nous dit que le degré du polynôme $P$ de degré inférieur ou égal à $r$ tel que $P(j)=c_{j}$ est donné par le minimum des $\ell$ tels que

$$
\Delta^{\ell}\left(c_{0}\right)=\Delta^{\ell}\left(c_{1}\right)=\cdots=\Delta^{\ell}\left(c_{r-\ell}\right) .
$$

Rappelons que les coefficients $a_{i j}$ sont déterminés par les relations ( $c f . \S 2$ )

$$
\sum_{i=0}^{r} \mathrm{X}^{i} a_{i j}=(\mathrm{X}-1)^{r-j}(\mathrm{X}+1)^{j}
$$

De ces relations il vient :

$$
\begin{aligned}
& \sum_{i=0}^{r} \mathrm{X}^{i} \Delta\left(a_{i j}\right)=\Delta\left((\mathrm{X}-1)^{r-j}(\mathrm{X}+1)^{j}\right)=2(\mathrm{X}-1)^{r-j-1}(\mathrm{X}+1)^{j} \\
& \sum_{i=0}^{r} \mathrm{X}^{i} \Delta^{\ell}\left(a_{i j}\right)=2^{\ell}(\mathrm{X}-1)^{r-j-\ell}(\mathrm{X}+1)^{j} .
\end{aligned}
$$

Donc, si $i>r-\ell$, on a $\Delta^{\ell}\left(a_{i j}\right)=0$ et si $\ell=r-i$, on a $\Delta^{\ell}\left(a_{i j}\right)=2^{\ell}$ pour $j=0, \ldots, r-\ell$; cela donne le résultat souhaité si $k$ est pair. Si $k$ est impair, il suffit de remarquer que $(-1)^{j} a_{i j}=(-1)^{r} a_{r-i j}$ (remplacer $X$ par $X^{-1}$ dans les relations qui déterminent les coefficients $a_{i j}$ ). 
On suppose maintenant que $k$ appartienne à l'intervalle $[1, n / r[$. On remarque qu'en $-k(k<n / r)$ les deux distributions $A(., s)$ et $A^{U}(., s)$ présente un pôle de même ordre : cela résulte d'un passage en coordonnés polaires (comme dans la remarque 2.1) et du fait que les supports des distributions $A^{h}(.,-k)$ soit des réunions de $G^{1}$ orbites. On peut alors procéder par récurrence sur le rang $r$ (le cas $r=1$ étant bien connu). Utilisant le corollaire 3.2 , il vient

$$
\Phi^{*} A^{U}(., s)=x_{-}^{s+d(r-1)} \otimes \mathrm{d} z \otimes A_{-}^{\prime}(., s)+x_{+}^{s+d(r-1)} \otimes \mathrm{d} z \otimes A_{+}^{\prime}(., s)
$$

avec :

$$
A_{-}^{\prime}(., s)=\sum_{i=0}^{r-1} c_{i+1} \Phi_{i}^{\prime}(., s), \quad A_{+}^{\prime}(., s)=\sum_{i=0}^{r-1} c_{i} \Phi_{i}^{\prime}(., s)
$$

Nous sommes ramenés à étudier en $s=-k$ les deux distributions $A_{-}^{\prime}(., s)$ et $A_{+}^{\prime}(., s)$ auxquelles on applique la récurrence. Soient $P_{-}$et $P_{+}$les deux polynômes de degré inférieur ou égal à $r-1$ tels que :

$$
\begin{array}{ll}
P_{-}(i)=c_{i+1}(-1)^{k i}, & i=0, \ldots, r-1, \\
P_{+}(i)=c_{i}(-1)^{k i}, & i=0, \ldots, r-1 .
\end{array}
$$

On a :

$$
\begin{array}{ll}
P_{+}(i)=P(i), & i=0, \ldots, r-1, \\
P_{-}(i)=(-1)^{k} P(i+1), & i=0, \ldots, r-1 .
\end{array}
$$

- Si $\mathrm{d}^{\circ} P \leq r-1$, on a

$$
P_{+}(\mathrm{X})=P(\mathrm{X}), \quad P_{-}(\mathrm{X})=(-1)^{k} P(\mathrm{X}+1)
$$

et donc les deux distributions $A_{-}^{\prime}(., s)$ et $A_{+}^{\prime}(., s)$ possèdent au point $s=-k$, par l'hypothèse de récurrence, un pôle d'ordre $\min \left(\mathrm{d}^{\circ} P, o^{\prime}(-k)\right)$; $o^{\prime}(-k)$ désigne l'ordre du pôle $-k$ pour la fonction

$$
\Gamma_{\Omega^{\prime}}\left(s+\left(\frac{n}{r}\right)^{\prime}\right)=(2 \pi)^{\frac{1}{2}\left(n^{\prime}-r^{\prime}\right)} \prod_{j=0}^{r^{\prime}-1} \Gamma\left(s-\frac{1}{2} j d\right)
$$

(on constate facilement que $d^{\prime}=d$ ), et donc pour $-k>-n / r$ on a $o(-k)=o^{\prime}(-k)$. Dans ce cas, la distribution $A(., s)$ possède bien en $s=-k$ un pôle d'ordre $\min \left(\mathrm{d}^{\circ} P, o(-k)\right)$.

$$
\text { TOME } 125-1997-\mathrm{N}^{\circ} 4
$$


- Reste le cas $\mathrm{d}^{\circ} P=r$. Montrons qu'alors un des deux polynômes $P_{-}$ ou $P_{+}$est de degré $r-1$; supposons en effet que $P_{-}$et $P_{+}$soient de degré inférieur ou égal à $r-2$, comme les deux polynômes (de degré inférieur ou égal à $r-2) P_{+}(X)$ et $(-1)^{k} P_{-}(X-1)$ coïncident en les $r-1$ points $i=1,2, \ldots, r-1$, ils sont égaux et par conséquent on a l'égalité :

$$
P_{+}(r)=(-1)^{k} P_{-}(r-1)=P(r) ;
$$

mais alors les deux polynômes $P_{+}$et $P$ coïncident en les $r+1$ points $i=0,2, \ldots, r$, ce qui est contradictoire avec la définition de $P$. Supposons par exemple que le polynôme $P_{+}$soit de degré $r-1$; la distribution $A_{+}^{\prime}(., s)$ présente alors par l'hypothèse de récurrence un pôle d'ordre $\min \left(r-1, o^{\prime}(-k)\right)=o^{\prime}(-k)=o(-k)=\min (r, o(-k))$ : cela achève la démonstration.

L'application du théorème 3.3 aux distributions $\Psi_{i}(., s)$ donne les résultats suivants :

Proposition 3.5.-Les distributions $\Psi_{i}(., s)$ présentent aux points $-2 k$ (resp. $-2 k-1)$ un pôle d'ordre

$$
\min (r-i, o(-2 k)) \quad(\text { resp. } \min (i, o(-2 k-1))) .
$$

En particulier, la distribution $|\operatorname{det} x|^{s}=\Psi_{r}(., s)$ est régulière en tous les entiers $-2 k$, et la distribution $|\operatorname{det} x|^{s} \operatorname{sign}(\operatorname{det} x)=(-1)^{r} \Psi_{0}(., s)$ est régulière en tous les entiers $-2 k-1$.

Rappelons que pour une distribution $A(., s)=\sum_{j=0}^{r} c_{j} \Phi_{j}(., s)$ de pôle effectif $s_{0}$ d'ordre $\alpha$, nous avons noté $\sum_{h=1}^{h=\alpha} A^{h}\left(., s_{0}\right) /\left(s-s_{0}\right)^{h}$ la partie singulière de son développement de Laurent au voisinage de ce pôle.

Donnons des précisions sur le support des distributions $A^{h}(.,-k)$. Suivant F. Ricci et E. Stein, nous dirons que le support d'une distribution est de rang $p$ s'il contient au moins un élément de rang $p$ mais aucun élément de rang strictement supérieur à $p$.

THÉORÈME 3.6. - Pour tout entier $k$ supérieur à 1 , et tout entier $h$ tel que $1 \leq h \leq \alpha$, le support de la distribution $A^{h}(.,-k)$ est de rang $r-h$.

En particulier, pour $k \geq n / r$, les distributions $B_{j}^{r}(.,-k)$ sont portées par $\{0\}$.

On procède par récurrence sur le rang $r$ de l'algèbre $V$ en utilisant la relation :

$$
\Phi^{*} A^{U}(., s)=x_{-}^{s+d(r-1)} \otimes \mathrm{d} z \otimes A_{-}^{\prime}(., s)+x_{+}^{s+d(r-1)} \otimes \mathrm{d} z \otimes A_{+}^{\prime}(., s) .
$$


- Supposons d'abord $\alpha=r$; puisque $\alpha=\min \left(\mathrm{d}^{\circ} P, o(-k)\right)$, on a $\mathrm{d}^{\circ} P=o(-k)=r$; considérant $\Phi^{*} A^{U}(., s)$, on voit que la distribution $A^{U}(., s)$ présente en $s=-k$ un pôle d'ordre inférieur ou égal à $r-1$ et par conséquent la restriction à $U$ de la distribution $A^{\alpha}(.,-k)$ est nulle. Comme $\{0\}$ est la seule $G^{1}$-orbite contenue dans $\left\{x_{11}=0\right\}$, il en résulte que le support de la distribution $A^{\alpha}(.,-k)$ est $\{0\}$. Regardons la distribution $\Phi^{*} A^{U}(., s)$ en $s=-k$; comme $\mathrm{d}^{\circ} P=r$, on montre, comme dans la démonstration du théorème 3.3 , qu'un des polynomes $P_{-}$et $P_{+}$ est de degré $r-1$.

Donc l'une des distributions $A_{-}^{\prime}(., s)$ et $A_{+}^{\prime}(., s)$ présente en $s=-k$ un pôle d'ordre $\min \left(r-1, o^{\prime}(-k)\right)$; mais $o(-k)=r$, donc $o^{\prime}(-k)=r-1$. La distribution $A^{U}(., s)$ présente donc en $s=-k$ un pôle d'ordre $r-1$. On regarde alors le développement de Laurent de $\Phi^{*} A^{U}(., s)$ en $s=-k$ et on obtient le résultat par récurrence (il ne peut y avoir d'élimination entre les deux distributions $x_{-}^{s+d(r-1)} \otimes \mathrm{d} z \otimes A_{-}^{\prime}(., s)$ et $\left.x_{+}^{s+d(r-1)} \otimes \mathrm{d} z \otimes A_{+}^{\prime}(., s)\right)$.

- Supposons maintenant $\alpha<r$. On a donc $\mathrm{d}^{\circ} P \leq r-1$; considérons à nouveau la distribution $\Phi^{*} A^{U}(., s)$ en $s=-k$; on a, comme dans la démonstration du théorème 3.3 ,

$$
P_{+}(X)=P(X) \quad \text { et } \quad P_{-}(X)=(-1)^{k} P(X+1)
$$

on en déduit que les deux distributions $A_{-}^{\prime}(., s)$ et $A_{+}^{\prime}(., s)$ présentent en $s=-k$ un pôle d'ordre $\min \left(\mathrm{d}^{\circ} P, o^{\prime}(-k)\right)=\alpha$ (on a soit $o^{\prime}(-k)=o(-k)$ soit $\left.o^{\prime}(-k)=o(-k)-1=r-1\right)$. On obtient le résultat par récurrence en regardant le développement de Laurent de $\Phi^{*} A^{U}(., s)$ en $s=-k$.

Donnons pour terminer ce paragraphe, l'analogue du corollaire 3 de [Ri-St].

Corollaire 3.7. - Soient $1 \leq p \leq r-1$ et $0 \leq q \leq p$. Pour tout entier $k$ tel que $r-p \leq o(-k)$, il existe une distribution $G$-homogène de degré $-k$, de support de rang $p$ et contenant l'orbite $S_{p, q}$.

On considère la distribution

$$
A(., s)=\sum_{j=0}^{r}(-1)^{k j} j^{r-p} \Phi_{j}(., s) .
$$

Par le théorème 3.3 , cette distribution présente en $s=-k$ un pôle d'ordre $r-p$ puisque $r-p \leq o(-k)$. Par le théorème 3.6, la distribution $A^{r-p}(.,-k)$ est à support de rang $p$. On va montrer par récurrence que le support de $A^{r-p}(.,-k)$ contient toutes les orbites de rang $p$.

TOME $125-1997-\mathrm{N}^{\circ} 4$ 
Pour $r=2$, on a $A(., s)=(-1)^{k} \Phi_{1}(., s)+2 \Phi_{2}(., s)$ (ici $k \geq 1$ et $p=1$ ). Appliquons la relation

$$
\Phi^{*} A^{U}(., s)=x_{-}^{s+d(r-1)} \otimes \mathrm{d} z \otimes A_{-}^{\prime}(., s)+x_{+}^{s+d(r-1)} \otimes \mathrm{d} z \otimes A_{+}^{\prime}(., s) .
$$

On a ici $A_{+}^{\prime}(., s)=(-1)^{k} x_{-}^{s}$ et $A_{-}^{\prime}(., s)=(-1)^{k} x_{+}^{s}+2 x_{-}^{s}$ et il est facile d'en conclure que le support de la distribution $A^{1}(.,-k)$ contient les deux orbites $S_{1,0}$ et $S_{1,1}$.

Plaçons-nous maintenant au rang $r$ et considérons à nouveau la distribution $\Phi^{*} A^{U}(., s)$; on a :

$$
\begin{aligned}
& A_{+}^{\prime}(., s)=\sum_{j=0}^{r-1}(-1)^{k j} j^{r-p} \Phi_{j}^{\prime}(., s), \\
& A_{-}^{\prime}(., s)=\sum_{j=0}^{r-1}(-1)^{k(j+1)}(j+1)^{r-p} \Phi_{j}^{\prime}(., s) .
\end{aligned}
$$

On a $r-p \leq r-1$ et $o^{\prime}(-k)=o(-k)$ ou $o^{\prime}(-k)=o(-k)-1=r-1$; par conséquent, d'après le théorème 3.3 , la distribution $A_{+}^{\prime}(., s)$ présente en $s=-k$ un pôle d'ordre $r-p$ et d'après le théorème 3.6 , la distribution $A_{+}^{\prime r-p}(.,-k)$ a un support de rang $r-1-p$. D'autre part, on peut écrire la distribution $A_{-}^{\prime}(., s)$ sous la forme

$$
A_{-}^{\prime}(., s)=(-1)^{k} A_{+}^{\prime}(., s)+B(., s)
$$

où $B(., s)$ est de la forme

$$
B(., s)=\sum_{j=0}^{r-1}(-1)^{k j} P(j) \Phi_{j}^{\prime}(., s)
$$

avec $P$ de degré strictement inférieur à $r-p$. La distribution $A_{-}^{\prime}(., s)$ présente donc en $s=-k$ un pôle d'ordre $r-p$ et $A_{-}^{\prime r-p}(.,-k)=$ $(-1)^{k} A_{+}^{\prime r-p}(.,-k)$; on peut alors conclure facilement par récurrence.

Remarque 3.3. - Par ce type de démonstration, on peut décrire entièrement le support des distributions $B_{j}^{h}(.,-k)$ : cela a été fait dans le cas où $V$ est l'algèbre $\operatorname{Herm}(r, \mathbb{C})$ des matrices hermitiennes par A. Chaabouni [Ch] (résultat non publié); M. Muro, Zürich, 1994, donne également ces supports. D'autre part on peut déterminer les relations linéaires reliant les différentes distributions $B_{j}^{h}(.,-k)$. 


\section{Les distributions homogènes sur l'algèbre $V$, le cas $d$ pair}

Nous sommes maintenant en mesure de donner des analogues des formules de I.M. Gelfand et G.E. Chilov évoquées dans l'introduction. Comme au paragraphe 3 , nous nous plaçons dans le cas où l'entier $d$ est pair, renvoyant le lecteur au paragraphe 5 pour le cas plus technique où l'entier $d$ est impair.

Reprenons les distributions $\Psi_{\ell}(., s)(0 \leq \ell \leq r)$, et posons :

$$
F_{\ell}(., s)= \begin{cases}a_{\ell}(s) \Psi_{\ell}(., s) 2^{-\frac{1}{2} r s} & \text { dans le cas }(\mathrm{i}), \\ a_{\ell}(s) \Psi_{\ell}(., s) 2^{-\frac{1}{2} r s-\frac{1}{2} \ell} & \text { dans le cas }(\mathrm{p}),\end{cases}
$$

avec :

$$
a_{\ell}(s)=\frac{\prod_{\substack{k \equiv 0 \\ k<n / r}}(s+k)^{\min (o(-k), r-\ell)} \prod_{\substack{k \equiv 1 \\ k<n / r}}(s+k)^{\min (o(-k), \ell)}}{\Gamma\left(\frac{1}{2}(s+\alpha)\right)^{r-\ell} \cdot \Gamma\left(\frac{1}{2}(s+\beta)\right)^{\ell}}
$$

où $\alpha$ est le plus petit entier pair supérieur à $n / r$, et $\beta$ le plus petit entier impair supérieur à $n / r$.

Par la proposition 3.5, les distributions $F_{\ell}(., s)$ sont des fonctions entières qui ne s'annulent jamais (les coefficients $a_{\ell}(s)$ ont été choisis à cet effet).

Notons $\epsilon_{\ell}$ le nombre qui vaut 1 si la somme des ordres des pôles strictement plus grand que $-n / r$ de $\Psi_{\ell}(., s)$ est pair et -1 sinon.

Proposition 4.1. - Les transformées de Fourier des distributions $F_{\ell}(., s)$ sont données par les formules:

$$
\begin{aligned}
& \widehat{F}_{\ell}(., s-n / r)=(2 \pi)^{\frac{1}{2} n}(-i)^{n} i^{\ell} \epsilon_{\ell} F_{\ell}(.,-s) \quad \text { dans le cas }(i), \\
& \widehat{F}_{\ell}(., s-n / r)=(2 \pi)^{\frac{1}{2} n}(-i)^{n} i^{\ell} \epsilon_{r-\ell} F_{r-\ell}(.,-s) \text { dans le cas }(p) \text {. }
\end{aligned}
$$

Remarque 4.1. - Pour $r=1$, cette proposition nous redonne les formules de I.M. Gelfand et G.E. Chilov.

On part des formules $(c f . \S 1)$ :

$$
\begin{aligned}
& \widehat{\Psi}_{\ell}(., s)=\left\{\begin{array}{l}
\gamma\left(s+\frac{n}{r}\right)(1+q)^{r-\ell}(1-q)^{\ell} \Psi_{\ell}\left(.,-s-\frac{n}{r}\right) \\
\gamma\left(s+\frac{n}{r}\right)(1+q)^{r-\ell}(1-q)^{\ell} \Psi_{r-\ell}\left(.,-s-\frac{n}{r}\right)
\end{array}\right. \text { dans le cas (i), } \\
& \text { томе } 125-1997-\mathrm{N}^{\circ} 4
\end{aligned}
$$


On a

$$
(1+q)^{r-\ell}(1-q)^{\ell}=\exp \left(\frac{1}{2} i r \pi s\right) 2^{r}(-i)^{\ell}\left(\cos \frac{1}{2} \pi s\right)^{r-\ell}\left(\sin \frac{1}{2} \pi s\right)^{\ell} .
$$

D'autre part,

$$
\Gamma_{\Omega}\left(s+\frac{n}{r}\right)=(2 \pi)^{\frac{1}{2}(n-r)}(\Gamma(s))^{r} \prod_{k=0}^{\frac{1}{2}(r-1) d}(s+k)^{r-o(-k)} .
$$

Notons, pour simplifier, $b_{\ell}(s)$ l'expression $\gamma\left(s+\frac{n}{r}\right)(1+q)^{r-\ell}(1-q)^{\ell}$; en utilisant les formules classiques :

$$
\begin{aligned}
\cos \frac{1}{2} \pi s & =\frac{\pi}{\Gamma\left(\frac{1}{2}(s+1)\right) \cdot \Gamma\left(\frac{1}{2}(1-s)\right)} \\
\sin \frac{1}{2} \pi s & =\frac{\pi}{\Gamma\left(\frac{1}{2} s\right) \cdot \Gamma\left(1-\frac{1}{2} s\right)} \\
\Gamma(s) & =\frac{2^{s-1}}{\sqrt{\pi}} \Gamma\left(\frac{1}{2} s\right) \cdot \Gamma\left(\frac{1}{2}(s+1)\right)
\end{aligned}
$$

il vient :

$$
\begin{aligned}
b_{\ell}(s)=(-i)^{\ell+n}(\pi)^{\frac{1}{2} n} 2^{\frac{1}{2}(n-r)} 2^{r s} & \\
& \times \frac{\prod_{k=0}^{\frac{1}{2}(r-1) d}(s+k)^{r-o(-k)} \Gamma\left(\frac{1}{2}(s+1)\right)^{\ell} \Gamma\left(\frac{1}{2} s\right)^{r-\ell}}{\Gamma\left(1-\frac{1}{2} s\right)^{\ell} \cdot \Gamma\left(\frac{1}{2}(1-s)\right)^{r-\ell}} .
\end{aligned}
$$

On écrit alors dans le membre de droite :

$$
\begin{aligned}
\left(\Gamma\left(\frac{1}{2} s\right) s\right)^{r-\ell} & =2^{r-\ell} \Gamma\left(\frac{1}{2}(s+2)\right)^{r-\ell} \\
\left(\Gamma\left(\frac{1}{2}(s+2)\right)(s+2)\right)^{r-\ell} & =2^{r-\ell} \Gamma\left(\frac{1}{2}(s+4)\right)^{r-\ell}
\end{aligned}
$$

etc., puis de même :

$$
\begin{aligned}
& \left(\Gamma\left(\frac{1}{2}(s+1)\right)(s+1)\right)^{\ell}=2^{\ell} \Gamma\left(\frac{1}{2}(s+3)\right)^{\ell}, \\
& \left(\Gamma\left(\frac{1}{2}(s+3)\right)(s+3)\right)^{\ell}=2^{\ell} \Gamma\left(\frac{1}{2}(s+5)\right),
\end{aligned}
$$

etc., ce qui nous donne :

$$
\begin{gathered}
b_{\ell}(s)=K_{\ell} \frac{\Gamma\left(\frac{1}{2}(s+\alpha)\right)^{r-\ell} \Gamma\left(\frac{1}{2}(s+\beta)\right)^{\ell}}{\Gamma\left(-\frac{1}{2} s\right)^{\ell} \Gamma\left(\frac{1}{2}(1-s)\right)^{r-\ell}} \\
\times \prod_{\substack{k \equiv 0 \\
1<k<n / r}}(s+k)^{\ell-o(-k)} \prod_{\substack{k \equiv 1 \\
k<n / r}}(s+k)^{r-o(-k)-\ell}
\end{gathered}
$$

BULLETIN DE LA SOCIÉTÉ MATHÉMATIQUE DE FRANCE 
avec :

$$
K_{\ell}= \begin{cases}(2 \pi)^{\frac{1}{2} n}(-1)^{n}(i)^{\ell+n} 2^{\frac{1}{2} n} 2^{r s} & \text { dans le cas (i) } \\ (2 \pi)^{\frac{1}{2} n}(-1)^{n}(i)^{\ell+n} 2^{\frac{1}{2} n+\ell-\frac{1}{2} r} 2^{r s} & \text { dans le cas (p) }\end{cases}
$$

Il vient ensuite :

$$
a_{\ell}(s) b_{\ell}(s)=K_{\ell} \frac{\prod_{\substack{k \equiv 0 \\ 0<k<n / r}}(s+k)^{\min (r-o(-k), \ell)} \prod_{\substack{k \equiv 1 \\ k<n / r}}(s+k)^{\min (r-o(-k), r-\ell)}}{\Gamma\left(-\frac{1}{2} s\right)^{\ell} \cdot \Gamma\left(\frac{1}{2}(1-s)\right)^{r-\ell}} .
$$

On remarque enfin que pour $0 \leq k \leq n / r$ on a $r-o(-k)=o(k-n / r)$, ce qui nous permet de comparer l'expression $a_{\ell}(s) b_{\ell}(s)$ à $a_{\ell}(-s-n / r)$ dans le cas (i), à $a_{r-\ell}(-s-n / r)$ dans le cas $(\mathrm{p})$, et d'en déduire la proposition.

ThÉORÈme 4.2. - La famille $\mathcal{F}(s)=\left\{F_{\ell}(., s) ; \ell=0, . ., r\right\}$ est, pour tout $s$, une base de l'espace $\mathcal{H}^{s}$ des distributions $G$-homogènes de degré s.

Comme la dimension de l'espace $\mathcal{H}^{s}$ est $r+1(c f .[\mathrm{Mu}]$, voir aussi $\S 6)$, il suffit de démontrer l'indépendance linéaire de la famille $\mathcal{F}(s)$.

La famille des distributions $\Psi_{\ell}(., s)$ étant libre pour $s$ n'appartenant pas à $-\mathbb{N}^{*}$, il en est de même pour $\mathcal{F}(s)$; d'autre part, par application de la transformée de Fourier, il suffit de montrer que la famille $\mathcal{F}(-k)$ est libre pour tous les entiers $k$ compris entre 1 et $n / r-1$.

Considérons $\mathcal{F}(-2 k)$. Soit

$$
\sum_{\ell \geq 0} \alpha_{\ell} F_{\ell}(.,-2 k)=0
$$

une combinaison linéaire reliant les distributions $F_{\ell}(.,-2 k)$; la distribution $F_{r}(.,-2 k)$ est portée par $V$ tout entier (car $\Psi_{r}(., s)$ est holomorphe au point $-2 k)$, tandis que les autres distributions $F_{\ell}(.,-2 k)$ sont à support dans le lieu singulier, on a donc $\alpha_{r}=0$; d'autre part, pour $\ell=0, \ldots, r-1$,

$$
F_{\ell}(.,-2 k)=c_{\ell} \sum_{j \geq 0} a_{\ell j} B_{j}^{\inf (o(-2 k), r-\ell)}(.,-2 k), \quad c_{\ell} \neq 0 .
$$

La relation $(*)$ devient alors :

$$
\begin{aligned}
\sum_{\ell \geq 0}^{r-o(-2 k)} \alpha_{\ell} c_{\ell}\left(\sum_{j \geq 0} a_{\ell j} B_{j}^{o(-2 k)}(.,-2 k)\right) & \\
+\sum_{\ell=r-o(-2 k)+1}^{r-1} \alpha_{\ell} c_{\ell}\left(\sum_{j \geq 0} a_{\ell j} B_{j}^{r-\ell}(.,-2 k)\right) & =0 .
\end{aligned}
$$

TOME $125-1997-\mathrm{N}^{\circ} 4$ 
Par application du théorème 3.6, on déduit les relations suivantes :

$$
\alpha_{\ell} c_{\ell}\left(\sum_{j \geq 0} a_{\ell j} B_{j}^{r-\ell}(.,-2 k)\right)=0
$$

pour $\ell=r-o(-2 k)+1, \ldots, r-1$ et

$$
\sum_{\ell \geq 0}^{r-o(-2 k)} \alpha_{\ell} c_{\ell}\left(\sum_{j \geq 0} a_{\ell j} B_{j}^{o(-2 k)}(.,-2 k)\right)=0 .
$$

Les premières équations nous donnent

$$
\alpha_{\ell}=0 \text { pour } \ell=r-o(-2 k)+1, \ldots, r-1
$$

puisque les sommes $\sum_{j \geq 0} a_{\ell j} B_{j}^{r-\ell}(.,-2 k)$ sont non nulles (les distributions $\sum_{j \geq 0} a_{\ell j} \Phi_{j}(., s)$ possèdent en $s=-2 k$ un pôle d'ordre $\left.r-\ell\right)$. La dernière relation s'écrit :

$$
\sum_{j \geq 0} \gamma_{j} B_{j}^{o(-2 k)}(.,-2 k)=0 \quad\left(\gamma_{j}=\sum_{\ell \geq 0}^{r-o(-2 k)} \alpha_{\ell} c_{\ell} a_{\ell j}\right)
$$

La distribution $\sum_{j \geq 0} \gamma_{j} \Phi_{j}(., s)$ admet, en $s=-2 k$, un pôle d'ordre $\min \left(\mathrm{d}^{\circ} Q, o(-2 k)\right)$ où $Q$ est le polynôme $\sum_{\ell \geq 0}^{r-o(-2 k)} \alpha_{\ell} c_{\ell} P_{\ell}$; comme on a $o(-2 k) \leq \mathrm{d}^{\circ} P_{\ell} \leq r$ pour $\ell$ compris entre 0 et $r-o(-2 k)$, il est facile d'en conclure $\alpha_{\ell}=0$ pour $\ell=0, \ldots, r-o(-2 k)$. La famille $\mathcal{F}(-2 k)$ est donc libre. On montre pareillement que la famille $\mathcal{F}(-2 k-1)$ est libre.

\section{Le cas $d$ impair}

Dans ce paragraphe, nous nous plaçons dans le cas où l'entier $d$ est impair, et énoncons les résultats analogues à ceux démontrés dans les paragraphes 3 et 4 . Toutes les démonstrations, quoique techniquement plus longues, sont essentiellement les mêmes que pour le cas où l'entier $d$ est pair. Notons d'autre part que nos résultats ne sont nouveaux que dans le cas de l'algèbre $V=\operatorname{Sym}(r, \mathbb{R})(d=1)$, le cas de l'algèbre $V=\mathbb{R} \times E$ étant classique ( $c f$. [Ge-Ch]).

On commence par revenir à la proposition 2.2 ; on a maintenant :

$$
P_{k}(\mathrm{X})= \begin{cases}\left(1-\mathrm{X}^{2}\right)^{\frac{1}{2} k} & \text { si } k \text { est pair } \\ \left(1-\mathrm{X}^{2}\right)^{\frac{1}{2}(k-1)}(1+\mathrm{X}) & \text { sinon. }\end{cases}
$$


Si l'on calcule les sommes $\sum_{j \equiv 0} \mathrm{X}^{j} u_{j k}(s)$ et $\sum_{j \equiv 1} \mathrm{X}^{j} u_{j k}(s)$ pour $k$ pair et pour $k$ impair, on constate que l'on peut imiter la méthode de [Sa-Fa] et interpréter les quatre matrices

$$
\begin{array}{ll}
\left(u_{j k}(s)\right)_{j \equiv 0, k \equiv 0}, & \left(u_{j k}(s)\right)_{j \equiv 0, k \equiv 1}, \\
\left(u_{j k}(s)\right)_{j \equiv 1, k \equiv 0}, & \left(u_{j k}(s)\right)_{j \equiv 1, k \equiv 1}
\end{array}
$$

en termes de représentations du groupe $\mathrm{GL}_{2}(\mathbb{R})$ comme dans le cas où l'entier $d$ est pair. Les résultats que l'on obtient sont les suivants :

- Cas $r$ impair et $k$ pair :

$$
\begin{aligned}
\sum_{j \equiv 0[2]} \mathrm{X}^{j} u_{j k}(s) & =\left(1-\mathrm{X}^{2} q^{2}\right)^{\frac{1}{2}(r-1)}\left(\frac{q^{2}-\mathrm{X}^{2}}{1-\mathrm{X}^{2} q^{2}}\right)^{\frac{1}{2} k} \\
\sum_{j \equiv 1[2]} \mathrm{X}^{j-1} u_{j k}(s) & =(-1)^{\frac{1}{2}(r-1)} q\left(1-\mathrm{X}^{2} q^{2}\right)^{\frac{1}{2}(r-1)}\left(\frac{q^{2}-\mathrm{X}^{2}}{1-\mathrm{X}^{2} q^{2}}\right)^{\frac{1}{2} k} .
\end{aligned}
$$

- Cas $r$ impair et $k$ impair :

$$
\begin{gathered}
\sum_{j \equiv 0[2]} \mathrm{X}^{j} u_{j k}(s)=q\left(1-\mathrm{X}^{2} q^{2}\right)^{\frac{1}{2}(r-1)}\left(\frac{q^{2}-\mathrm{X}^{2}}{1-\mathrm{X}^{2} q^{2}}\right)^{\frac{1}{2}(k-1)} \\
\sum_{j \equiv 1[2]} \mathrm{X}^{j-1} u_{j k}(s)=(-1)^{\frac{1}{2}(r-1)}\left(1-\mathrm{X}^{2} q^{2}\right)^{\frac{1}{2}(r-1)}\left(\frac{q^{2}-\mathrm{X}^{2}}{1-\mathrm{X}^{2} q^{2}}\right)^{\frac{1}{2}(k-1)} .
\end{gathered}
$$

(Rappelons que $q=\exp (i \pi s)$.)

Indiquons brièvement comment interpréter les matrices $\left(u_{j k}(s)\right)$.

Soit $\mathcal{P}_{r, p}$ l'espace des polynômes à une variable, pairs, de degré inférieur ou égal à $r$. Pour un élément $f$ de $\mathcal{P}_{r, p}$, on note $\tilde{f}$ le polynôme de degré inférieur ou égal à $\frac{1}{2}(r-1)$ défini par

$$
\tilde{f}\left(\mathrm{X}^{2}\right)=f(\mathrm{X}) .
$$

On définit une représentation $\rho_{r, p}$ de $\mathrm{GL}_{2}(\mathbb{C})$ dans l'espace $\mathcal{P}_{r, p}$ par

$$
\rho_{r, p}\left(\begin{array}{ll}
a & b \\
c & d
\end{array}\right) f=g
$$

avec :

$$
\tilde{g}(\mathrm{X})=(b \mathrm{X}+d)^{\frac{1}{2}(r-1)} \tilde{f}\left(\frac{a \mathrm{X}+c}{b \mathrm{X}+d}\right)=\rho_{\frac{1}{2}(r-1)}\left(\begin{array}{ll}
a & b \\
c & d
\end{array}\right) \tilde{f}
$$

TOME $125-1997-\mathrm{N}^{\circ} 4$ 
On a alors :

$$
\rho_{r, p}\left(\begin{array}{cc}
-1 & -q^{2} \\
q^{2} & 1
\end{array}\right) X^{k}=\left(1-\mathrm{X}^{2} q^{2}\right)^{\frac{r-1}{2}}\left(\frac{q^{2}-\mathrm{X}^{2}}{1-\mathrm{X}^{2} q^{2}}\right)^{\frac{1}{2} k}
$$

ce qui permet d'interpréter les deux matrices

$$
\left(u_{j k}(s)\right)_{j \equiv 0, k \equiv 0} \text { et }\left(u_{j k}(s)\right)_{j \equiv 1, k \equiv 0} .
$$

On procède d'une manière analogue pour les deux autres matrices

$$
\left(u_{j k}(s)\right)_{j \equiv 0, k \equiv 1} \text { et }\left(u_{j k}(s)\right)_{j \equiv 1, k \equiv 1} .
$$

On détermine alors, comme dans [Sa-Fa], une famille de distributions $\left\{\Psi_{\ell}^{ \pm}(., s) ; \ell \equiv 0[2] ; 0 \leq \ell \leq r-1\right\}$ :

$$
\Psi_{\ell}^{ \pm}(., s)=\sum_{j \equiv 0} a_{\ell j}\left(\Phi_{j}(., s) \pm \Phi_{j+1}(., s)\right),
$$

où les coefficients $a_{\ell j}$ sont donnés par les relations

$$
\sum_{\ell \equiv 0}^{r} \mathrm{X}^{\ell} a_{\ell j}=\left(\mathrm{X}^{2}-1\right)^{\frac{1}{2}(r-1-j)}\left(\mathrm{X}^{2}+1\right)^{\frac{1}{2} j}
$$

avec :

$$
\widehat{\Psi_{\ell}^{ \pm}}\left(., s-\frac{n}{r}\right)= \begin{cases}\gamma(s) c_{\ell}(s)(1 \pm q) \Psi_{r-1-\ell}^{ \pm}(.,-s) & \text { si } n / r \equiv 1 \bmod 2, \\ \gamma(s) c_{\ell}(s)(1 \mp q) \Psi_{r-1-\ell}^{\mp}(.,-s) & \text { si } n / r \equiv 0 \bmod 2,\end{cases}
$$

et $c_{\ell}(s)=(-1)^{\frac{1}{2}(r-1-\ell)}\left(1+q^{2}\right)^{\frac{1}{2}(r-1-\ell)}\left(q^{2}-1\right)^{\frac{1}{2} \ell}$.

- Cas $r$ pair et $k$ pair :

$$
\begin{aligned}
& \sum_{j \equiv 0[2]} \mathrm{X}^{j} u_{j k}(s)=\left(1+\mathrm{X}^{2} q^{2}\right)^{\frac{1}{2} r}\left(\frac{q^{2}+\mathrm{X}^{2}}{1+\mathrm{X}^{2} q^{2}}\right)^{\frac{1}{2} k}, \\
& \sum_{j \equiv 1[2]} \mathrm{X}^{j} u_{j k}(s)=0 .
\end{aligned}
$$

- Cas $r$ pair et $k$ impair :

$$
\begin{aligned}
\sum_{j \equiv 0[2]} \mathrm{X}^{j} u_{j k}(s) & =q\left(1+\mathrm{X}^{2} q^{2}\right)^{\frac{1}{2} r-1}\left(\frac{q^{2}+\mathrm{X}^{2}}{1+\mathrm{X}^{2} q^{2}}\right)^{\frac{1}{2}(k-1)}\left(1+\mathrm{X}^{2}\right), \\
\sum_{j \equiv 1[2]} \mathrm{X}^{j-1} u_{j k}(s) & =-2 q \cos \pi s\left(1+\mathrm{X}^{2} q^{2}\right)^{\frac{1}{2} r-1}\left(\frac{q^{2}+\mathrm{X}^{2}}{1+\mathrm{X}^{2} q^{2}}\right)^{\frac{1}{2}(k-1)} .
\end{aligned}
$$

BULLETIN DE LA SOCIÉTÉ MATHÉMATIQUE DE FRANCE 
À nouveau on détermine, par une analyse analogue à celle faite dans le cas $r$ impair, une famille de distributions $\left\{\Psi_{\ell}^{+}(., s) ; \ell \equiv 0[2], 0 \leq \ell \leq r\right\}$, $\left.\Psi_{\ell}^{-}(., s) ; \ell \equiv 0[2], 0 \leq \ell \leq r-2\right\}$ :

$$
\Psi_{\ell}^{+}(., s)=\sum_{j \equiv 0} a_{\ell j}^{+} \Phi_{j}(., s) ; \quad \Psi_{\ell}^{-}(., s)=\sum_{j \equiv 0} a_{\ell j}^{-} \Phi_{j+1}(., s),
$$

où les coefficients $a_{\ell j}^{+}$et $a_{\ell j}^{-}$sont donnés par les relations :

$$
\begin{aligned}
& \sum_{\ell \equiv 0}^{r} \mathrm{X}^{\ell} a_{\ell j}^{+}=\left(\mathrm{X}^{2}-1\right)^{\frac{1}{2}(r-j)}\left(\mathrm{X}^{2}+1\right)^{\frac{1}{2} j}, \\
& \sum_{\ell \equiv 0}^{r-2} \mathrm{X}^{\ell} a_{\ell j}^{-}=\left(\mathrm{X}^{2}-1\right)^{\frac{1}{2}(r-2-j)}\left(\mathrm{X}^{2}+1\right)^{\frac{1}{2} j},
\end{aligned}
$$

avec :

$$
\begin{aligned}
& \widehat{\Psi_{\ell}^{-}}\left(., s-\frac{n}{r}\right)=\gamma(s) i^{d}(-1)^{\frac{1}{2} r+1} d_{\ell}(s) \Psi_{\ell}^{-}(.,-s), \\
& \widehat{\Psi_{\ell}^{+}}\left(., s-\frac{n}{r}\right)=\gamma(s) d_{\ell}(s)\left(\Psi_{\ell}^{+}(.,-s)+\frac{2 q}{\left(1+q^{2}\right)} \Psi_{\ell-2}^{-}(.,-s)\right),
\end{aligned}
$$

avec

$$
d_{\ell}(s)=\left(1-q^{2}\right)^{\frac{1}{2}(r-\ell)}\left(1+q^{2}\right)^{\frac{1}{2} \ell},
$$

pour $\ell$ pair, compris entre 0 et $r$ et la convention $\Psi_{-2}^{-}(., s)=0$. On constate alors que si l'on pose

$$
\Psi_{\ell}^{\prime}(., s)=\Psi_{\ell}^{+}(., s)+\cos \pi s \Psi_{\ell-2}^{-}(., s),
$$

on a :

$$
\widehat{\Psi_{\ell}^{\prime}}\left(., s-\frac{n}{r}\right)=\gamma(s) d_{\ell}(s) \Psi_{\ell}^{\prime}(.,-s) .
$$

\section{A. Généralisation des résultats de F. Ricci et E.M. Stein, le cas $d$ impair.}

Considérons maintenant, comme dans le paragraphe 3 , une distribution $A(., s)$ de la forme $A(., s)=\sum_{j=0}^{r} c_{j} \Phi_{j}(., s)$. Il y a deux séries de pôles possibles pour cette distribution : les entiers négatifs plus petits que -1 , et les demi-entiers négatifs plus petits que $-\frac{3}{2}$; à partir d'un tel pôle $s_{0}$, et des coefficients $c_{i}$ de la distribution $A(., s)$, construisons deux polynômes $P_{0}$ et $P_{1}$ tels que :

$$
\begin{cases}c_{j} \exp \left(i \pi j s_{0}\right)=P_{0}(j), \mathrm{d}^{\circ} P_{0} \leq\left[\frac{1}{2} r\right] & \text { si } j \text { est pair, } \\ c_{j} \exp \left(i \pi j s_{0}\right)=P_{1}(j), \mathrm{d}^{\circ} P_{1} \leq\left[\frac{1}{2}(r-1)\right] & \text { si } j \text { est impair. }\end{cases}
$$

TOME $125-1997-\mathrm{N}^{\circ} 4$ 
Posons par ailleurs

$$
\epsilon= \begin{cases}\epsilon\left(P_{0}, P_{1}\right)=1 & \text { si d } \mathrm{d}^{\circ}\left(P_{o}-P_{1}\right)=\sup \left(\mathrm{d}^{\circ} P_{o}, \mathrm{~d}^{\circ} P_{1}\right) \\ 0 & \text { sinon. }\end{cases}
$$

On a les résultats suivants :

THÉORÈME 5.1 .

1) En $s=-k-\frac{1}{2}$, la distibution $A(., s)$ possède un pôle d'ordre

$$
\min \left(\sup \left(\mathrm{d}^{\circ} P_{o}, \mathrm{~d}^{\circ} P_{1}\right), o\left(-k-\frac{1}{2}\right)\right)
$$

2) En $s=-k$, la distribution $A(., s)$ possède un pôle d'ordre

$$
\min \left(\sup \left(\mathrm{d}^{\circ} P_{o}, \mathrm{~d}^{\circ} P_{1}\right)+\epsilon, o(-k)\right) .
$$

La démonstration, quoique techniquement plus compliquée, est analogue à celle du théorème 3.3 , et ne contient aucune idée nouvelle : pour les pôles inférieurs ou égaux à $-n / r$, il suffit d'établir le résultat pour les distributions $\Psi_{k}^{ \pm}(., s)$ (dans le cas où l'entier $r$ est pair, on peut s'aider pour cela des distributions $\Psi_{k}^{\prime}(., s)$ ), et pour les autres pôles, on procède par récurrence.

Ici l'application du théorème 5.1 aux distributions $\Phi_{j}(., s)$ donne dans le cas où l'entier $r$ est impair, le corollaire suivant :

Corollaire 5.2. - On suppose $r$ impair. Pour tous les pôles $s_{0}$ de la fonction $\Gamma_{\Omega}(s+n / r)$ et tous les $j(0 \leq j \leq r)$, on a $o_{j}\left(s_{0}\right)=o\left(s_{0}\right)$.

Dans le cas où l'entier $r$ est pair, la situation est plus compliquée :

Corollaire 5.3. - Supposons $r$ pair; alors:

1) pour tout $j(0 \leq j \leq r)$ et tout $k$ entier supérieur à 1 , on a $o_{j}(-k)=o(-k)$

2) pour tout $j$ pair $(0 \leq j \leq r)$ et tout $k$ entier supérieur $\grave{a} 1$, on a $o_{j}\left(-k-\frac{1}{2}\right)=o\left(-k-\frac{1}{2}\right)$;

3) pour tout $j$ impair $(0 \leq j \leq r)$, on a $o_{j}\left(-k-\frac{1}{2}\right)=o\left(-k-\frac{1}{2}\right)-\eta$ où $\eta=0$ si $k$ est compris entre 1 et $\frac{1}{2}(r-2)$ et $\eta=1$ si $k$ est supérieur où égal à $\frac{1}{2} r$.

Notons que ce phénomène de "perte de multiplicité» avait déjà été constaté dans [Ge-Ch] à propos de l'algèbre $\mathbb{R} \times E$.

En ce qui concerne le rang du support des distributions $A^{h}\left(., s_{0}\right)$, on a les résultats suivants :

BULLETIN DE LA SOCIÉTÉ MATHÉMATIQUE DE FRANCE 
ThÉORÈme 5.4. - Soit $s_{0}$ un pôle effectif entier de la distribution $A(., s)$ d'ordre $\alpha$. Pour tout $h$ compris entre 1 et $\alpha$, le support de la distribution $A^{h}\left(., s_{0}\right)$ est de rang $r+1-2 h$ si $s_{0}$ est un entier, et de rang $r-2 h$ si $s_{0}$ est un demi-entier. En particulier, pour $r$ impair (resp. $r$ pair) et pour $k \geq n / r$, les distributions $B_{j}^{\frac{1}{2}(r+1)}(.,-k)\left(B_{j}^{\frac{1}{2} r}\left(.,-k-\frac{1}{2}\right), j\right.$ pair $)$ sont portées par $\{0\}$.

La démonstration suit celle du théorème 3.6.

Enfin, donnons l'analogue du corollaire 3 de [Ri-St] :

Corollaire 5.5. - Soient $1 \leq p \leq r-1$ et $0 \leq q \leq p$. Supposons $p$ de la forme $r+1-2 h$ avec $h$ un entier; alors pour tout entier $k$ tel que $h \leq o(-k)$, il existe une distribution $G$-homogène de degré $-k$, de support de rang $p$ et contenant l'orbite $S_{p, q}$. Supposons $p$ de la forme $r-2 h$ avec $h$ un entier; alors pour tout demi-entier $k+\frac{1}{2}$ tel que $h \leq o\left(-k-\frac{1}{2}\right)$, il existe une distribution $G$-homogène de degré $-k-\frac{1}{2}$, de support de rang $p$ et contenant l'orbite $S_{p, q}$.

Supposons $p$ de la forme $r+1-2 h$ avec $h$ un entier et soit $k$ un entier tel que $h \leq o(-k)$; on considère les distributions

$$
\begin{aligned}
& A(., s)=\sum_{j \equiv 0[2]} \exp (i \pi k) j^{h-1} \Phi_{j}(., s), \\
& B(., s)=\sum_{j \equiv 1[2]} \exp (i \pi k) j^{h-1} \Phi_{j}(., s) .
\end{aligned}
$$

Par le théorème 5.1 , ces distributions présentent en $s=-k$ un pôle d'ordre $h$ puisque $h \leq o(-k)$, et par le théorème 5.4, les distributions $A^{h}(.,-k)$ et $B^{h}(.,-k)$ sont à support de rang $r+1-2 h=p$. En procédant comme dans la démonstration du corollaire 3.7 , on montre par récurrence sur les deux distributions que leur support contient toutes les orbites de rang $p$.

Supposons $p$ de la forme $r-2 h$ avec $h$ un entier et soit $s_{0}$ un demi-entier tel que $h \leq o\left(s_{0}\right)$; on considère les distributions

$$
\begin{aligned}
& A(., s)=\sum_{j \equiv 0[2]} \exp \left(-i \pi s_{0}\right) j^{h} \Phi_{j}(., s), \\
& B(., s)=\sum_{j \equiv 1[2]} \exp \left(-i \pi s_{0}\right) j^{h} \Phi_{j}(., s) .
\end{aligned}
$$

À nouveau, par le théorème 5.1, ces distributions présentent en $s=s_{0}$ un pôle d'ordre $h$ et par le théorème 5.4 , les distributions $A^{h}\left(., s_{0}\right)$ et $B^{h}\left(., s_{0}\right)$ sont à support de rang $r-2 h=p$, mais cette fois ci leur support 
a une structure plus compliquée : on montre par récurrence sur les deux distributions que le support de $A^{h}\left(., s_{0}\right)$ contient toutes les orbites $S_{p, q}$ avec $q$ pair, et que celui de $B^{h}\left(., s_{0}\right)$ contient toutes les orbites $S_{p, q}$ avec $q$ impair.

\section{B. Les distributions homogènes sur l'algèbre $V$, le cas $d$ impair.}

On peut encore normaliser les distributions $\Psi_{\ell}^{ \pm}(., s)$ dans le cas où l'entier $r$ est impair, et les distributions $\Psi_{\ell}^{+}(., s), \Psi_{\ell}^{\prime}(., s)$ si $r$ est pair, et obtenir une famille holomorphe de distributions $G$-homogènes :

- Cas $r$ impair.

Dans le cas où l'entier $n / r$ est impair, on pose pour $\ell$ pair et compris entre 0 et $r-1$ :

$$
F_{\ell}^{ \pm}(., s)=2^{-\frac{n}{2 r} s} \cdot 2^{\frac{1}{8}(r-3) \ell} \sqrt{\pi}^{-\frac{1}{2} \ell} a_{\ell}^{ \pm}(s) \Psi_{\ell}^{ \pm}(., s)
$$

avec :

$$
\begin{aligned}
& a_{\ell}^{ \pm}(s)=\frac{N}{D}, \\
& N=\prod_{\substack{k \equiv 0 \\
k<n / r}}(s+k)^{\min \left(k, \frac{1}{2}(r \mp 1-\ell)\right)} \\
& \times \prod_{\substack{k \equiv 1 \\
k<n / r}}(s+k)^{\min \left(k, \frac{1}{2}(r \pm 1-\ell)\right)} \\
& D=\Gamma\left(\frac{1}{2}(s+\alpha)\right)^{\frac{1}{2}(r \mp 1-\ell)} \cdot \Gamma\left(\frac{1}{2}(s+\beta)\right)^{\frac{1}{2}(r \pm 1-\ell)} \cdot \Gamma\left(s+\frac{1}{2} r\right)^{\frac{1}{2} \ell}
\end{aligned}
$$

où $\alpha$ est le plus petit entier pair supérieur à $n / r$, et $\beta$ le plus petit entier impair supérieur à $n / r$.

Dans le cas où l'entier $n / r$ est pair, on pose pour $\ell$ pair et compris entre 0 et $r-1$ :

$$
F_{\ell}^{ \pm}(., s)=2^{-\frac{n}{2 r} s} \cdot 2^{\frac{1}{8}(r-3) \ell} \sqrt{\pi}^{-\frac{1}{2} \ell} c^{ \pm} a_{\ell}^{ \pm}(s) \Psi_{\ell}^{ \pm}(., s)
$$

avec les mêmes coefficients $a_{\ell}^{ \pm}(s)$ et où $c^{+}$vaut 1 et $c^{-}$vaut $\sqrt{2}$.

Notons $\epsilon_{\ell}^{ \pm}$le nombre qui vaut 1 si la somme des ordres des pôles strictement plus grand que $-n / r$ de $\Psi_{\ell}^{ \pm}(., s)$ est pair et -1 sinon. Par 
des manipulations identiques à celles effectuées dans la proposition 4.1, on obtient les formules suivantes :

$$
\begin{aligned}
& \frac{n}{r} \equiv 1[2]\left\{\begin{array}{l}
\widehat{F}_{\ell}^{+}\left(., s-\frac{n}{r}\right)=(2 \pi)^{\frac{1}{2} n}(-i)^{n} i^{\frac{1}{2} \ell} i \epsilon_{r-1-\ell}^{+} F_{r-1-\ell}^{+}(.,-s), \\
\widehat{F}_{\ell}^{-}\left(., s-\frac{n}{r}\right)=(2 \pi)^{\frac{1}{2} n}(-i)^{n} i^{\frac{1}{2} \ell} \epsilon_{r-1-\ell}^{-} F_{r-1-\ell}^{-}(.,-s)
\end{array}\right. \\
& \frac{n}{r} \equiv 0[2]\left\{\begin{array}{l}
\widehat{F}_{\ell}^{+}\left(., s-\frac{n}{r}\right)=(2 \pi)^{\frac{1}{2} n}(-i)^{n} i^{\frac{1}{2} \ell} i \epsilon_{r-1-\ell}^{-} F_{r-1-\ell}^{-}(.,-s), \\
\widehat{F}_{\ell}^{-}\left(., s-\frac{n}{r}\right)=(2 \pi)^{\frac{1}{2} n}(-i)^{n} i^{\frac{1}{2} \ell} \epsilon_{r-1-\ell}^{+} F_{r-1-\ell}^{+}(.,-s) .
\end{array}\right.
\end{aligned}
$$

- Cas r pair. - On pose :

$F_{\ell}^{+}(., s)=a_{\ell}(s) \Psi_{\ell}^{\prime}(., s)$ pour $\ell$ pair et compris entre 0 et $r$,

$F_{\ell}^{-}(., s)=a_{\ell}(s) \Psi_{\ell}^{-}(., s)$ pour $\ell$ pair et compris entre 0 et $r-2$,

avec (un produit vide est interprété comme valant 1) :

$$
a_{\ell}(s)=\frac{\prod_{k=1}^{k<\frac{n}{r}-\frac{1}{2}}(s+k)^{\min \left(k, \frac{1}{2}(r-\ell)\right)} \prod_{k=1}^{k<\frac{n}{r}-\frac{1}{2}}\left(s+k+\frac{1}{2}\right)^{\min \left(k, \frac{1}{2} \ell\right)}}{\Gamma\left(s+\frac{n}{r}-\frac{1}{2}\right)^{\frac{1}{2}(r-\ell)} \Gamma\left(s+\frac{n}{r}\right)^{\frac{1}{2} \ell}}
$$

( $\ell$ pair et compris entre 0 et $r$ ).

On montre, grâce au théorème 5.1, que la famille des distributions $F_{\ell}^{ \pm}(., s)$ est une famille holomorphe sur $\mathbb{C}$ tout entier.

On note $\epsilon_{\ell}$ le nombre qui vaut 1 si la somme des ordres des pôles strictement plus grand que $-n / r$ de $\Psi_{\ell}^{\prime}(., s)$ est pair et -1 sinon. On obtient les formules :

$$
\widehat{F}_{\ell}^{-}\left(., s-\frac{n}{r}\right)=(2 \pi)^{\frac{1}{2} n} i^{\frac{1}{2}(r+\ell)+d}(-1)^{\frac{1}{2} r+1} \epsilon_{\ell} F_{\ell}^{-}(.,-s)
$$

( $\ell$ pair et compris entre 0 et $r-2$ ),

$$
\widehat{F}_{\ell}^{+}\left(., s-\frac{n}{r}\right)=(2 \pi)^{\frac{1}{2} n} i^{\frac{1}{2}(r+\ell)} \epsilon_{\ell} F_{\ell}^{+}(.,-s)
$$

( $\ell$ pair et compris entre 0 et $r$ ).

Dans les deux cas on a le théorème :

THÉORÈME 5.5. - La famille des $F_{\ell}^{ \pm}(., s)$ est une famille holomorphe sur $\mathbb{C}$ tout entier et est, pour tout $s$, une base de l'espace $\mathcal{H}^{s}$.

Cela résulte $\mathrm{du}$ théorème 5.1 et d'un raisonnement analogue à celui effectué pour la démonstration du théorème 4.3.

Donnons pour terminer quelques exemples. Pour simplifier, nous noterons $\Phi_{i}(s)$ la distribution $\Phi_{i}(., s)$.

$$
\text { TOME } 125-1997-\mathrm{N}^{\circ} 4
$$


- Cas $r=2$, $d$ pair. - Dans ce cas, l'algèbre $V$ est $\mathbb{R} \times \mathbb{R}^{d+1}$ et det $x$ est une forme quadratique de signature $(1, d+1)$. La dimension de l'espace $\mathcal{H}^{s}$ est 3.

On a dans le cas (i) :

$$
\begin{aligned}
& F_{0}(s)=2^{-s} a_{0}(s)\left(\Phi_{0}(s)-\Phi_{1}(s)+\Phi_{2}(s)\right), \\
& F_{1}(s)=2^{-s} a_{1}(s)\left(-2 \Phi_{0}(s)+2 \Phi_{2}(s)\right) \\
& F_{2}(s)=2^{-s} a_{2}(s)\left(\Phi_{0}(s)+\Phi_{1}(s)+\Phi_{2}(s)\right)
\end{aligned}
$$

avec :

$$
\begin{gathered}
a_{0}(s)=\frac{\prod_{k=1}^{\frac{1}{4} d}(s+2 k)}{\Gamma\left(\frac{1}{2} s+\frac{1}{4} d+1\right)^{2}}, \quad a_{2}(s)=\frac{\prod_{k \equiv 1}^{\frac{1}{2} d-1}(s+k)}{\Gamma\left(\frac{1}{2}(s+1)+\frac{1}{4} d\right)^{2}}, \\
\prod_{1}(s)=\frac{\prod_{k=1}^{\frac{1}{2} d}(s+k)}{\Gamma\left(\frac{1}{2} s+\frac{1}{4} d+1\right) \cdot \Gamma\left(\frac{1}{2}(s+1)+\frac{1}{4} d\right)}
\end{gathered}
$$

et dans le cas $(p)$ :

$$
\begin{aligned}
& F_{0}(s)=2^{-s} a_{0}(s)\left(\Phi_{0}(s)-\Phi_{1}(s)+\Phi_{2}(s)\right), \\
& F_{1}(s)=2^{-s-\frac{1}{2}} a_{1}(s)\left(-2 \Phi_{0}(s)+2 \Phi_{2}(s)\right), \\
& F_{2}(s)=2^{-s-1} a_{2}(s)\left(\Phi_{0}(s)+\Phi_{1}(s)+\Phi_{2}(s)\right)
\end{aligned}
$$

avec :

$$
\begin{gathered}
a_{0}(s)=\frac{\prod_{k=1}^{\frac{1}{4}(d-2)}(s+2 k)}{\Gamma\left(\frac{1}{2}(s+1)+\frac{1}{2} d\right)^{2}}, \quad a_{2}(s)=\frac{\prod_{k \equiv 1}^{\frac{1}{2} d}(s+k)}{\Gamma\left(\frac{1}{2} s+\frac{1}{4} d+1\right)^{2}} \\
a_{1}(s)=\frac{\prod_{k=1}^{\frac{1}{2} d}(s+k)}{\Gamma\left(\frac{1}{2}(s+1)+\frac{1}{4} d\right) \cdot \Gamma\left(\frac{1}{2} s+\frac{1}{4} d+1\right)}
\end{gathered}
$$

Posons $\vec{F}(s)=\left(F_{0}(s), F_{1}(s), F_{2}(s)\right)^{t} ;$ on a :

$$
\widehat{\vec{F}}\left(s-\frac{1}{2} n\right)=(2 \pi)^{\frac{1}{2} n} A \vec{F}(-s)
$$

BULLETIN DE LA SOCIÉTÉ MATHÉMATIQUE DE FRANCE 
avec

$$
\begin{aligned}
& A=\left(\begin{array}{ccc}
-(-1)^{\frac{1}{4}(n-2)} & 0 & 0 \\
0 & -i & 0 \\
0 & 0 & (-1)^{\frac{1}{4}(n-2)}
\end{array}\right) \quad \text { dans le cas (i) } \\
& A=\left(\begin{array}{ccc}
0 & 0 & (-1)^{\frac{1}{4} n} \\
0 & -i & 0 \\
(-1)^{\frac{1}{4} n} & 0 & 0
\end{array}\right) \quad \text { dans le cas }(\mathrm{p}) \text {. }
\end{aligned}
$$

- Cas $r=2$, $d$ impair. - Ici, $V$ est toujours égal à $\mathbb{R} \times \mathbb{R}^{d+1}$; on a :

$$
\begin{aligned}
& F_{0}^{+}(s)=a_{0}(s)\left(-\Phi_{0}(s)+\Phi_{2}(s)\right), \\
& F_{2}^{+}(s)=a_{2}(s)\left(\Phi_{0}(s)+\Phi_{2}(s)+\cos \pi s \Phi_{1}(s)\right), \\
& F_{0}^{-}(s)=a_{0}(s) \Phi_{1}(s),
\end{aligned}
$$

avec :

$$
a_{0}(s)=\frac{1}{\Gamma(s+1)}, \quad a_{2}(s)=\frac{1}{\Gamma\left(s+\frac{3}{2}\right)} .
$$

Posons $\vec{F}(s)=\left(F_{0}^{+}(s), F_{2}^{+}(s), F_{0}^{-}(s)\right)^{t}$; on a :

$$
\widehat{\vec{F}}\left(s-\frac{1}{2} n\right)=(2 \pi)^{\frac{1}{2} n} A \vec{F}(-s)
$$

avec

$$
A=\left(\begin{array}{ccc}
(-1)^{\frac{1}{2}(n-1)} i & 0 & 0 \\
0 & (-1)^{\frac{1}{2}(n-1)} & 0 \\
0 & 0 & 1
\end{array}\right)
$$

- Cas $r=3, d=1(V=\operatorname{Sym}(3, \mathbb{R}))$. - Ici la dimension de l'espace $\mathcal{H}^{s}$ est 4 . On a :

$$
\begin{aligned}
& F_{0}^{+}(s)=2^{-s} a_{0}^{+}(s)\left(-\Phi_{0}(s)-\Phi_{1}(s)+\Phi_{2}(s)+\Phi_{3}(s)\right), \\
& F_{2}^{+}(s)=2^{-s} \frac{1}{\sqrt{\pi}} a_{2}^{+}(s)\left(\Phi_{0}(s)+\Phi_{1}(s)+\Phi_{2}(s)+\Phi_{3}(s)\right), \\
& F_{0}^{-}(s)=2^{-s} \sqrt{2} a_{0}^{-}(s)\left(-\Phi_{0}(s)+\Phi_{1}(s)+\Phi_{2}(s)-\Phi_{3}(s)\right), \\
& F_{2}^{-}(s)=2^{-s} \frac{\sqrt{2}}{\sqrt{\pi}} a_{2}^{-}(s)\left(\Phi_{0}(s)-\Phi_{1}(s)+\Phi_{2}(s)-\Phi_{3}(s)\right),
\end{aligned}
$$

avec :

$$
\begin{array}{ll}
a_{0}^{+}(s)=\frac{s+1}{\Gamma\left(\frac{1}{2}(s+2)\right) \cdot \Gamma\left(\frac{1}{2}(s+3)\right)^{2}}, & a_{2}^{+}(s)=\frac{s+1}{\Gamma\left(\frac{1}{2}(s+3)\right) \cdot \Gamma\left(s+\frac{3}{2}\right)}, \\
a_{0}^{-}(s)=\frac{s+1}{\Gamma\left(\frac{1}{2}(s+2)\right)^{2} \cdot \Gamma\left(\frac{1}{2}(s+3)\right)}, & a_{2}^{-}(s)=\frac{1}{\Gamma\left(\frac{1}{2}(s+2)\right) \cdot \Gamma\left(s+\frac{3}{2}\right)} . \\
\text { TOME } 125-1997-\mathrm{N}^{\circ} 4 &
\end{array}
$$


Posons $\vec{F}(s)=\left(F_{0}^{+}(s), F_{2}^{+}(s), F_{0}^{-}(s), F_{2}^{-}(s)\right)^{t}$; on a :

$$
\widehat{\vec{F}}(s-2)=(2 \pi)^{3} A \vec{F}(-s), \quad A=\left(\begin{array}{cccc}
0 & 0 & 0 & i \\
0 & 0 & -1 & 0 \\
0 & -1 & 0 & 0 \\
i & 0 & 0 & 0
\end{array}\right) .
$$

- Cas $r=4, d=1(V=\operatorname{Sym}(4, \mathbb{R}))$. - Ici la dimension de l'espace $\mathcal{H}^{s}$ est 5 . On a :

$$
\begin{aligned}
& F_{0}^{+}(s)=a_{0}(s)\left(\Phi_{0}(s)-\Phi_{2}(s)+\Phi_{4}(s)\right), \\
& F_{2}^{+}(s)=a_{2}(s)\left(-2 \Phi_{0}(s)+2 \Phi_{4}(s)+\cos \pi s\left(-\Phi_{1}(s)+\Phi_{3}(s)\right)\right), \\
& F_{4}^{+}(s)=a_{4}(s)\left(\Phi_{0}(s)+\Phi_{2}(s)+\Phi_{4}(s)+\cos \pi s\left(\Phi_{1}(s)+\Phi_{3}(s)\right)\right), \\
& F_{0}^{-}(s)=a_{0}(s)\left(-\Phi_{1}(s)+\Phi_{3}(s)\right), \\
& F_{2}^{-}(s)=a_{2}(s)\left(\Phi_{1}(s)+\Phi_{3}(s)\right)
\end{aligned}
$$

avec :

$$
a_{0}(s)=\frac{s+1}{\Gamma(s+2)^{2}}, \quad a_{2}(s)=\frac{(s+1)\left(s+\frac{3}{2}\right)}{\Gamma(s+2) \Gamma\left(s+\frac{5}{2}\right)}, \quad a_{4}(s)=\frac{s+\frac{3}{2}}{\Gamma\left(s+\frac{5}{2}\right)^{2}} .
$$$$
\text { Posons } \vec{F}(s)=\left(F_{0}^{+}(s), F_{2}^{+}(s), F_{4}^{+}(s), F_{0}^{-}(s), F_{2}^{-}(s)\right)^{t} \text {; on a : }
$$

$$
\widehat{\vec{F}}\left(s-\frac{5}{2}\right)=(2 \pi)^{5} A \vec{F}(-s), \quad A=\left(\begin{array}{ccccc}
1 & 0 & 0 & 0 & 0 \\
0 & i & 0 & 0 & 0 \\
0 & 0 & -1 & 0 & 0 \\
0 & 0 & 0 & -i & 0 \\
0 & 0 & 0 & 0 & 1
\end{array}\right) .
$$

\section{Appendice}

Cet appendice est destiné à convaincre le lecteur que les méthodes de [Ri-St] pour déterminer la dimension de l'espace $\mathcal{H}^{s}$ se généralisent aux cas d'une algèbre simple et euclidienne $V$ et que l'on peut ainsi redémontrer le résultat de M. Muro.

\section{A. Généralités.}

Fixons un système complet d'idempotents orthogonaux $\left\{e_{1}, \ldots, e_{r}\right\}$ de $V$ et considérons la décomposition de Peirce associée à ce système :

$$
V=\bigoplus_{1 \leq i \leq j \leq r} V_{i j}
$$


Si $S_{p q}$ est une orbite singulière de rang $p$ de l'algèbre $V\left(S_{p, q}=G o_{p, q}\right.$ où

$$
o_{p, q}=\sum_{i=1}^{p-q} e_{i}-\sum_{i=1}^{q} e_{p-q+i}
$$

avec $0 \leq q \leq p$ et $p<r$ ), notons $T_{p q}$ (resp. $N_{p q}$ ) l'espace tangent (resp. l'espace normal) à $S_{p q}$ en $o_{p, q}$. On vérifie alors facilement que l'espace $N_{p q}$ s'identifie naturellement à :

tandis que $T_{p q}$ s'identifie à :

$$
\bigoplus_{p<i \leq j \leq r} V_{i j}
$$

$$
\bigoplus_{\substack{1 \leq i \leq j \leq r \\ i \leq p}} V_{i j}
$$

En particulier, $N_{p q}$ est une algèbre de Jordan simple et euclidienne de rang $r-p$ que nous noterons $V_{(p)}$. Dans la suite, si $O$ est un objet relatif à l'algèbre $V$, nous noterons $O_{(p)}$ l'objet correspondant pour l'algèbre $N_{p q}$; en particulier $\wp_{(p)}^{k}$ désigne l'espace des polynômes homogènes de degré $k$ sur l'algèbre $V_{(p)}$.

Notons $H_{p q}$ le sous groupe de $G$ qui laisse fixe le point $o_{p, q}$; on vérifie que les éléments de $A$ de la forme

$$
a=\mathcal{P}\left(o_{p, 0}+\sum_{i=p+1}^{r} a_{i} e_{i}\right)
$$

( $a_{i}$ positifs non nuls) appartiennent à $H_{p q}$ et laissent invariant $V_{(p)}$ et $T_{p q}$; la restriction d'un tel élément $a$ à l'algèbre $V_{(p)}$ est l'élément

$$
a_{(p)}=\mathcal{P}_{(p)}\left(\sum_{i=p+1}^{r} a_{i} e_{i}\right)
$$

du sous-groupe $A_{(p)}$ de $G_{(p)}$; on vérifie également la relation

$$
\operatorname{Det} a=\left(\operatorname{Det}_{(p)} a_{(p)}\right)\left(\frac{r}{n}\right)_{(p)} \frac{n}{r} \text {. }
$$

On calcule facilement le déterminant de l'action de $a$ sur l'espace tangent $T_{p q}$; on trouve :

$$
\prod_{i=p+1}^{r} a_{i}^{d p}=(\operatorname{Det} a)^{\frac{r d p}{2 n}}
$$

Soit d'autre part $z$ un élément de $V_{i j}$ avec $p<i<j \leq r$; on vérifie que $\exp \left(2 z \square e_{i}\right)$ est un élément de $H_{p q}$ qui laisse invariant $V_{(p)}$ et $T_{p q}$; la restriction de $\exp \left(2 z \square e_{i}\right)$ à l'algèbre $V_{(p)}$ est un élément du sousgroupe $N_{(p)}$ de $G_{(p)}$ (ces éléments engendrent le sous-groupe $N_{(p)}$ ). 
Posons $W_{p q}=\bigcup_{p^{\prime}=p}^{r} \bigcup_{q^{\prime}=q}^{q+p^{\prime}-p} S_{p^{\prime} q^{\prime}}$, c'est un voisinage ouvert de l'orbite $S_{p q}$, invariant sous $G$.

\section{B. Un lemme crucial.}

Nous dirons qu'une distribution $K$ est $G$ quasi-homogène de degré $s$ s'il existe des distributions $U_{1}, \ldots, U_{\ell}$ telles que pour tout $g$ dans $G$ :

$$
g \cdot K=(\operatorname{Det} g)^{\frac{r s}{n}+1} K+(\operatorname{Det} g)^{\frac{r s}{n}}+1 \sum_{i=1}^{\ell}(\log \operatorname{Det} g)^{i} U_{i}
$$

(où $g \cdot K(\phi)=K\left(\phi_{g}\right)$ si $\phi_{g}(x)=\phi\left(g^{-1} x\right)$ ). Un nombre complexe sera dit critique s'il est pôle de la fonction $\Gamma_{\Omega}(s+n / r)$.

Le lemme suivant donne des conditions sur l'existence de distributions quasi-homogènes à support dans le lieu singulier de $V$, c'est l'analogue du lemme 5 de [Ri-St] :

Lemme 6.1. - Soit $K$ une distribution $G$ quasi-homogène de degré $s$ à support de rang $p$ où $1 \leq p \leq r-1$. Soit $S_{p q}$ une $G$-orbite de rang $p$ dans l'algèbre $V$. Alors $s$ est critique et $s \leq-\left(\frac{n}{r}-\frac{1}{2} d p\right)$, la restriction de la distribution $K$ à $W_{p q}$ est une distribution $G$-homogène de degré s, et cette restiction est unique à une constante multiplicative près.

La démonstration consiste à copier celle donnée dans [Ri-St], nous ne donnerons que les changements principaux. On commence par supposer que $K$ est $G$-homogène, et on regarde la distribution $K$ localement autour du point $o_{p, q}$ en utilisant le théorème de Schwartz. On arrive à l'analogue de la formule (31), p. 158, de [Ri-St] :

$$
K=\sum_{m=0}^{M} \sum_{i_{1} \leq i_{2} \leq \ldots \leq i_{m}} Y_{i_{1}} \ldots Y_{i_{m}} \psi_{I}
$$

dans $W$ un voisinage adéquat de $o_{p, q}$ contenu dans $W_{p q}$, où $I=$ $\left(i_{1}, \ldots, i_{m}\right)$, les fonctions $\psi_{I}$ étant régulières sur $S_{p q} \cap W, Y_{i_{1}} \ldots Y_{i_{m}}$ sont des champs de vecteurs sur $W$ que l'on suppose engendrer en tout point de $S_{p q} \cap W$ l'espace normal à l'orbite $S_{p q}$, et où $M$ est l'ordre de transversalité de la distribution $K$.

Exprimant ensuite l'homogénéité de la distribution $K$, on arrive à :

$$
\psi_{I}(x)=(\operatorname{Det} g)^{-\frac{r s}{n}-1} J_{g}(x) \sum_{|L|=M} \gamma_{L I}(g, x) \psi_{L}(g \cdot x)
$$


pour $|I|=M$, où $|I|$ est l'ordre de $Y^{I}, g$ étant proche de l'identité, $J_{g}(x)$ est le jacobien de l'action de $g$ sur $S_{p q}$ et où $\gamma_{L I}(g, x)=\beta_{I L}\left(g^{-1}, x\right)$, les fonctions $\beta_{I L}(g, x)$ étant donnés par la relation (38) :

$$
g \tilde{Y}^{I}=\sum_{|J|=M} \beta_{J I}(g, x) \tilde{Y}^{J}+\sum_{|J|<M} \cdots
$$

( $\tilde{Y}^{I}$ désigne l'adjoint de $Y^{I}$ sur $V$ ).

Dans la relation (38), faisons $x=o_{p, q}$ et prenons $g$ dans $H_{p q}$ tel que son action laisse stable les espaces $T_{p q}$ et $N_{p q}$. Observons qu'alors l'action de $g$ sur $N_{p q}$ s'identifie à l'action naturelle de $\operatorname{GL}\left(V_{(p)}\right)$ sur $V_{(p)} ;$ soit $S^{M}\left(V_{(p)}\right)$ la $M$-ième puissance symétrique de $V_{(p)}$. La relation (38) entraîne alors que pour de tels $g$ la matrice $\beta_{I J}\left(g, o_{p, q}\right)$ est la matrice dans une certaine base de l'action naturelle de $g_{(p)}$ - où l'on note par $g_{(p)}$ la restriction de $g$ à $V_{(p)}$ - dans l'espace $S^{M}\left(V_{(p)}\right)$ (et donc la matrice $\gamma_{I J}\left(g, o_{p, q}\right)$ représente l'action contragrédiente de $g_{(p)}$ dans $\wp_{(p)}^{M}$ identifié au dual de $\left.S^{M}\left(V_{(p)}\right)\right)$. On constuit ensuite avec les nombres $\left(\psi_{I}\left(o_{p, q}\right)\right)_{|I|=M}$ un élément $v$ de $\wp_{(p)}^{M}$ tel que (où l'action d'un élément $h$ de $\operatorname{GL}\left(V_{(p)}\right)$ sur $v$ est donné par $\left.h \cdot v(x)=v\left(h^{-1} x\right)\right)$ :

$$
g_{(p)} \cdot v=(\operatorname{Det} g)^{\frac{r s}{n}+1}\left(J_{g}(x)\right)^{-1} v
$$

pour $g$ dans $H_{p q}$ et tel que son action laisse stable les espaces $T_{p q}$ et $N_{p q}$ on peut en particulier prendre pour $g$ les éléments $\mathcal{P}\left(o_{p, 0}+\sum_{i=p+1}^{r} a_{i} e_{i}\right)$ et $\exp \left(2 z \square e_{i}\right)$ évoqués dans les généralités, on en déduit alors que le polynôme $v$ est conique au sens de [Fa-Ko, p. 224]); en décomposant $\wp_{(p)}^{M}$ en $G_{(p)}$ modules irréductibles, on voit que $v$ est nécessairement de la forme $\left(\operatorname{det}_{(p)} y\right)^{k}$ ( à une constante multiplicative près) où $k$ est un entier positif et que l'on a la relation $s=-k-\left(\frac{n}{r}-\frac{1}{2} d p\right)$. Cela nous donne donc le résultat d'unicité pour $K$ du corollaire ainsi que la relation pour le nombre complexe $s$.

Pour le cas où $K$ est quasi-homogène, on suit sans problèmes les raisonnements de [Ri-St] en appliquant les corollaires 3.7 et 5.5 au lieu de leur corollaire 3 .

On peut maintenant montrer que la dimension de l'espace $\mathcal{H}^{s}$ est égal au nombre d'orbites ouvertes : pour $s$ non critique, on prouve qu'une distribution $T$, supposée $G$-homogène de degré $s$, est une combinaison linéaire des $\Phi_{i}(., s)$; pour $s=s_{0}$ critique considérons dans le cas où $d$

TOME $125-1997-\mathrm{N}^{\circ} 4$ 
est pair les distributions $A_{m}^{s}=\sum_{q=0}^{r}(-1)^{s_{0} q} q^{m} \Phi_{q}(., s)(0 \leq m \leq r)$ : par le théorème 3.3 , ces distributions présentent en $s=s_{0}$ un pôle d'ordre $\min \left(m, o\left(s_{0}\right)\right)$ et on montre par application du lemme 6.1 que $T$ est combinaison linéaire $\operatorname{des} A^{\min \left(m, o\left(s_{0}\right)\right)}$; quand l'entier $d$ est impair, on remplace les distributions $A_{m}^{s}$ par les distributions $A_{i}\left(0 \leq i \leq E\left(\frac{1}{2} r\right)\right.$, $E(x)$ désignant la partie entière de $x)$ et $B_{i}\left(0 \leq i \leq E\left(\frac{1}{2}(r-1)\right)\right)$ données par :

$$
\begin{aligned}
& A_{i}=\sum_{q \equiv 0[2]} \exp \left(-i \pi q s_{0}\right) q^{i} \Phi_{q}(., s), \\
& B_{i}=\sum_{q \equiv 1[2]} \exp \left(-i \pi q s_{0}\right) q^{i} \Phi_{q}(., s) .
\end{aligned}
$$

\section{BIBLIOGRAPHIE}

[Br-Koe] Braun (H.), Koecher (M.). - Jordan Algebra. - Springer Verlag, 1966.

[Ch] Chanbouni (A.). - Propriétés des intégrales zeta associées à un espace préhomogène. Relations entre les fonctions moyennes sur l'espace des matrices hermitiennes. - Thèse, Université de Tunis, 1986.

[Fa-Ko] Faraut (J.), Koranyi (A.). - Analysis on symmetric cones. Clarendon Press, 1994.

[Gi] Gindikin (S.G.). - Analysis in Homogeneous domains, Russian. Math. Survays, t. 19, 1964, p. 1-80.

[Ge-Ch] Gelfand (I.M.), Chilov (G.E.). - Les distributions I. - Dunod, 1962.

[Ka] KAnEYUKI (S.). - On the causal structures of the Shilov boundaries of symmetric bounded domains. - Prospects in complex geometry, Proceedings, Katata/Kyoto, 1989, J. Noguchi, T. Ohsawa (eds), Lecture Notes in Math., 1468, Springer, p. 127-159.

[Mu] Muro (M.). - Invariant hyperfunctions on regular prehomogeneous vector spaces of commutative parabolic type, Tôhoku Math. J., t. 42, 1990, p. 163-193.

[Ra] RAï (M.). - Distributions homogènes sur des espaces de matrices, Bull. Soc. Math. France, Mémoire, 30, 1972.

[Ri-St] Ricci (F.), Stein (E.). - Homogeneous distributions on space of Hermitean matrices, J. für die reine und ang. Math., t. 368, 1986, p. 142-164. 
[Sa1] SATAKe (I.). - Algebraic structures of symmetric domains. Iwanami-Shoten and Princeton Univ. Press, 1980.

[Sa2] SATAKe (I.). - On zeta functions associated with self dual homogeneous cones. - Reports on Symposium of Geometry and Automorphic Functions, Tohoku Univ., Sendai, 1988.

[Sa-Fa] Satake (I.), Faraut (J.). - The functional equation of zeta distributions associated with formally real Jordan algebras, Tôhoku Math. J., t. 36, 1984, p. 469-482.

[Sa-Og] Satake (I.), Ogata (S.). - Zeta functions associated to cones and their special values, Advanced Studies in pure Math., t. 15, 1989, p. $1-27$.

[Sa-Sh] Sato (M.), Shintani (T.). - On zeta function associated with prehomogeneous vector spaces, Ann. of Math., t. 100, 1974, p. 131-170.

[We] WeIL (A.). - Fonction zêta et distributions, Séminaire Bourbaki, t. 12, 1966. 